(C) (c) 2015 IEEE. Personal use of this material is permitted. Permission from IEEE must be obtained for all other uses, in any current or future media, including reprinting/republishing this material for advertising or promotional purposes, creating new collective works, for resale or redistribution to servers or lists, or reuse of any copyrighted component of this work in other works. 


\title{
Needle-guiding Robot for Laser Ablation of Liver Tumors under MRI Guidance
}

\author{
E. Franco, D. Brujic, M. Rea, W. M. Gedroyc, M. Ristic, Member, IEEE
}

\begin{abstract}
This paper presents the design, control and experimental evaluation of a needle-guiding robot intended for use in laser ablation (LA) of liver tumors under guidance by Magnetic Resonance Imaging (MRI). The robot provides alignment of a needle guide inside the MRI scanner bore and employs manual needle insertion. In order to minimize MRimage deterioration, the robot is actuated using plastic pneumatic cylinders and long pipes connecting to control valves located outside the MRI scanner room. A new Time Delay Control scheme (TDC) was employed to achieve high position accuracy without requiring pressure or force measurements in the MRI scanner. The control scheme was compared with experiments to a previously developed Sliding Mode Controller (SMC). A marker localization method based on the convolution theorem of Fourier transform was employed to register the robot in the MRI scanner coordinate system and to verify the position of the needle guide before the manual needle insertion. Experiments in a closed-bore MRI scanner showed a variation in SNR below 5\%. A phantom study indicates that the targeting error in robot-assisted needle insertions is below $5 \mathbf{~ m m}$ and suggest a potential time saving of 30 minutes compared to the manual MRI-guided LA procedure.
\end{abstract}

Index Terms-Medical Robotics, Pneumatic System, MRI.

\section{INTRODUCTION}

$\mathrm{H}$ EPATOCELLULAR carcinoma (HCC) is the third leading cause of cancer-related death worldwide and its incidence is continually rising as a result of the spread of hepatitis $\mathrm{C}$ virus infection [1]. Approximately, $30 \%$ to $40 \%$ of cases present early tumors that can be treated with resection, liver transplantation, or percutaneous ablation [2]. In practice, $90 \%$ of patients are not suitable for resection due to lesion location, multiple lesions or poor liver function, while liver transplantation is limited by the shortage of donor organs [3]. Laser Ablation (LA) is used to treat otherwise inoperable liver tumors by delivering thermal energy from a high power laser directly to the target lesions [4]. Thermal energy is delivered through water-cooled high power sheaths which are placed using a coaxial puncture needle [5]. Compared to conventional ultrasound-guided procedures, Magnetic Resonance Imaging (MRI) guidance offers better soft tissue contrast, reducing the

This work was supported by the i4i Grant II-AR-1109-11011. E.Franco, D. Brujic and M. Ristic are with the Mechanical Engineering Department, Imperial College London (e-mail: ef1311@imperial.ac.uk, d.brujic@imperia.ac.uk, m.ristic@imperial.ac.uk).

M. Rea is with the Institute of Clinical Science, MRI Department, St Mary's Campus (e-mail: marc.rea@imperial.ac.uk).

W. M. Gedroyc is with the Department of Medicine, MRI Unit, St Mary's Campus (email: w.gedroyc@imperial.ac.uk). risk of puncturing vital organs and blood vessels, while also allowing thermal monitoring [6]. Open MRI magnets have been successfully employed for real time imaging of needle placement [6]. However, they are less common compared to closed-bore MRI scanners and their lower field and gradient strength reduce image quality and increase scan time [5]. Closed-bore MRI scanners are commonly available in most hospitals, but the patient access is severely restricted. Hence, placing the needle under MRI-guidance requires a complex and time-consuming workflow which involves repeatedly moving the patient bed in and out of the scanner. As a result, the duration of the procedure can exceed 2 hours while the ablation therapy typically takes 30 minutes [1].

A fully functional needle-guiding robot for microwave thermotherapy of liver tumors was presented in [7]. The robot has 3 actuated orthogonal prismatic joints $(150 \mathrm{~mm}$ travel) and two manual inclinations of the needle guide $\left( \pm 40^{\circ}\right.$ and $\pm 60^{\circ}$ range). However, inside closed-bore MRI scanners, the needle guide cannot be accessed by the clinicians. Consequently, setting the two inclinations would require moving the patient bed out of the scanner as in the manual procedure. A new prototype designed for closed-bore MRI scanners was presented in [8], however it was still manually actuated.

The aim of this research is the development of a remotely controlled needle-guiding robot that will operate in closedbore MRI scanners in order to reduce the procedure duration and to improve the targeting accuracy in LA of the liver. The main technical challenges in the robot development are preserving the quality of the MR images and achieving accurate positioning. The MRI environment limits the actuation methods allowed for robotic devices; therefore, manual actuation, ultrasonic motors, pneumatic and hydraulic cylinders represent the most common choices [9]. Ultrasonic motors have been widely employed in medical robots since they produce high torques and are not back-drivable. However, considerable losses in signal-to-noise ratio (SNR) were reported when actuating the motors in the MRI scanner [7], [10]-[12]. Nevertheless, incremental improvements have been obtained employing RF shielding [11], while negligible image degradation was experienced when keeping the ultrasonic motors outside the imaging volume [13]. Recently, the successful use of piezoelectric actuators was demonstrated in [14], [15] with a novel motor driver, achieving a reduction in SNR below $15 \%$. New piezoelectric actuators have recently been proposed [16], offering some potential for the future, although at present they are still limited to small-range 
movements. Pneumatic actuation has been successfully used in robots for MRI-guided intervention [17]-[20], since it produces minimum alterations to the MRI environment and, compared to hydraulics, it is clean and inexpensive. However, pneumatic valves should be placed far from the scanner, since they employ electric currents that can interact with the magnetic field. In some notable solutions this issue was addressed placing the valves inside a special shielded enclosure in the scanner room [17], [18]. In other works it was considered advantageous placing the valves outside the scanner room [12], [20], [21]. While the first approach reduces the length of the pipes, the second requires a simpler design and limits the use of electric components in the scanner room. However, the consequent use of long supply lines introduces delays which limit the bandwidth of the transmission. Additionally, commercially available pressure and force sensor, which are typically not MRI-safe [29], cannot be placed inside the scanner bore. Therefore, the ability to measure and compensate loads acting on the piston is restricted, adversely affecting the performance.

Previously proposed solutions aiming at achieving accurate positioning might be summarized as follows. Timing belts were used in a parallel robot for prostate biopsy [17] to increase the damping of the cylinders in order to reduce oscillations and improve stability. Sufficient accuracy was achieved at the cost of slower response and higher design complexity. Special high-viscous-friction cylinders were successfully employed in a 6-DOF robot for the treatment of sciatic pain [18]. However, the cylinders proved sensitive to disturbances and small temperature changes. Pneumatic stepper motors were recently employed in a robot for endorectal prostate biopsy [19] achieving high targeting accuracy. The main drawback of this solution is the complex construction of the motors. Additionally, the motors are naturally suited to produce rotary motion, while additional transmissions would be required for linear movements, with inherent issues of friction and mechanical backlash. Recently, a new stepper motor design was proposed in [22], which offers smaller size and simpler construction at the cost of reduced power and limited positioning accuracy. The most common control approaches for direct-drive pneumatic actuations with long supply lines employed in the MRI environment are PID schemes [17], [18], [20] and Sliding Mode Control (SMC) schemes, such as [23]-[25] and references therein. Recently, higher accuracy was achieved with a Time Delay Controller (TDC) in [26], which however did not account for the pressure dynamics due to valves and pipes.

The 4-DOF needle-guiding robot presented in this paper (Fig. 1) employs commercially available plastic pneumatic cylinders and is remotely actuated from outside the MRI scanner room through long pipes. The robot builds upon our previous 3-DOF proof-of-concept [25], while offering several important new features. Firstly, the additional actuated rotation of the needle guide allows double-inclined needle insertions and larger workspace. Secondly, accurate position control of the pistons is achieved with a new TDC scheme that takes into account the pressure dynamics due to valves and pipes. The

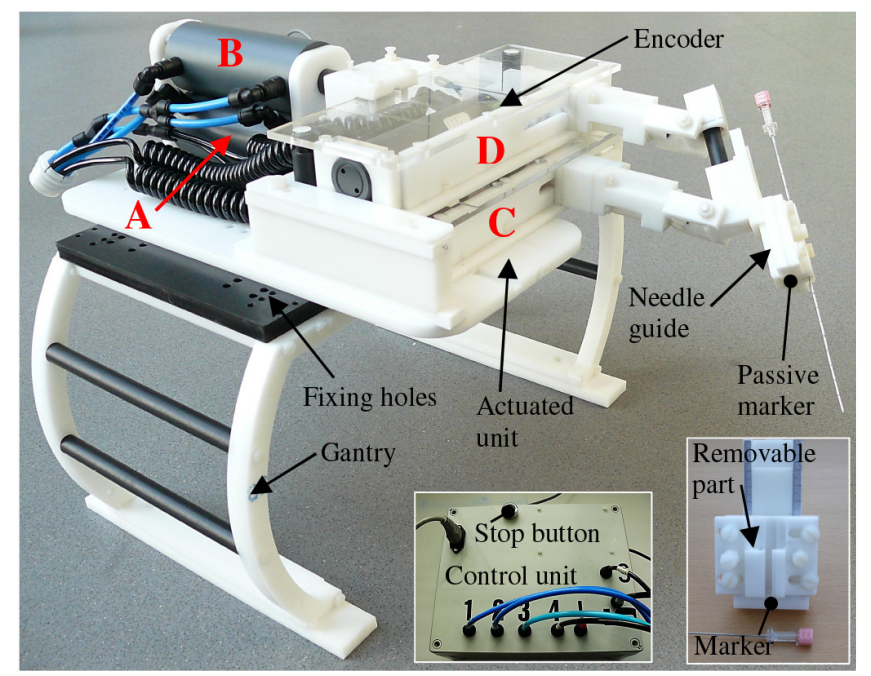

Fig. 1. Prototype of the needle-guiding robot (cylinders are A, B, C, D).

controller achieves steady-state errors smaller than $0.05 \mathrm{~mm}$ without relying on pressure or force measurements in the MRI scanner. Finally, a MR image processing method and passive fiducial markers are used to register the robot in the MRI scanner. Instead, our first prototype [25] employed semi-active markers and special imaging sequences which have to be reprogrammed for different MRI scanners.

This paper is organized as follows. Section II presents the workflow of the clinical procedure and defines the design requirements. Section III describes robot's kinematics, workspace, and design. Section IV presents the controller design. Section $\mathrm{V}$ gives an overview of the marker localization method. Section VI reports experimental results of MRIcompatibility tests, motion tests, and targeting accuracy tests on a phantom. Section VII contains concluding remarks.

\section{Procedure WorkFlow AND SySTEM REQUIREMENTS}

The emphasis in the development of the needle-guiding robot is to streamline the most time-consuming and errorprone phases of the LA procedure while minimizing disruptions to the established clinical workflow. Part A of this section describes the workflow of the LA procedure, while part B outlines the system requirements.

\section{A. Workflow of MRI-guided LA}

The workflow of MRI-guided LA of liver tumors is depicted in Fig. 2 which also reports the typical duration of each phase, based on direct observation. Initially, the patient is laid supine on the scanner bed with a receiver coil placed on the abdomen. The approximate position of the lesions within the patient's liver is known from pre-operative MR images. After an initial phase which involves preparing the equipment and the patient, new MR images are acquired to locate the target lesion and to establish the optimal needle insertion path. Placing the coaxial needle under MRI-guidance in a closed-bore scanner can involve several scans and iterations. Alternatively, the coaxial needle and the ablation probe can be inserted under ultrasound guidance at a different site, after which the patient is moved to the MRI scanner [6]. This approach is equally time-consuming 


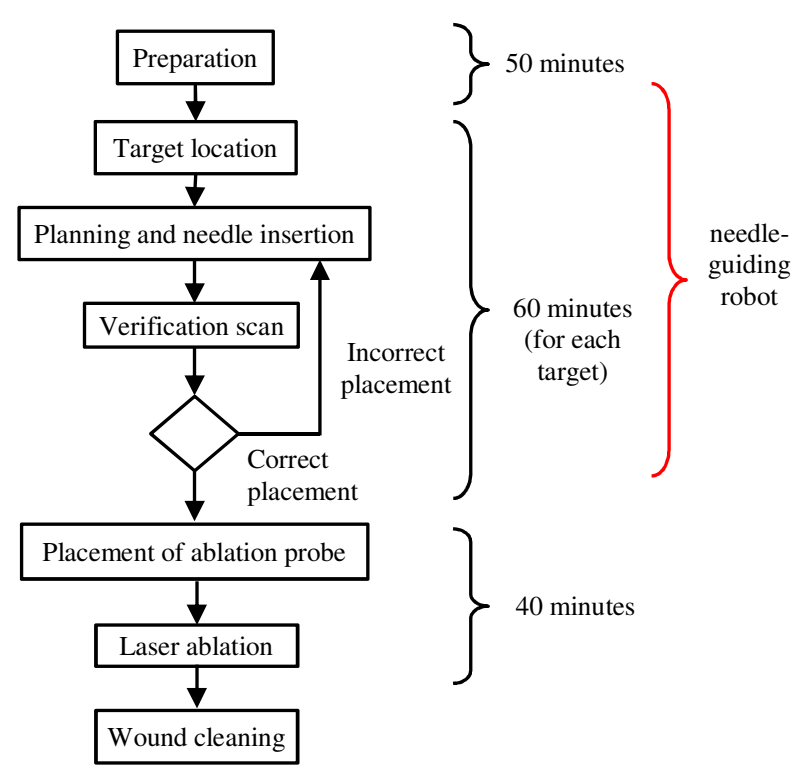

Fig. 2. Workflow of MRI-guided LA of liver tumors and typical duration.

and bears the risk of dislodging the probe while moving the patient. Once the coaxial needle is in place, the ablation probe is inserted and the ablation procedure is carried out. Finally, the probe is removed and the wound is cleaned.

It is apparent from Fig. 2 that the central phase of the procedure is the most time-consuming and that it would benefit the most from a robotic assistant. The improvements achieved by the proposed system are indicated in Fig. 13.

\section{B. System Requirements}

The requirements for the needle-guiding robot were defined in close collaboration with clinicians and were based on the relevant norms. The clinical requirements are: 1) plan needle insertions quickly and accurately; 2) provide visual verification on the MR images prior to the needle insertion; 3) achieve a targeting accuracy sufficient for clinically relevant lesions; 4) target lesions in the whole liver.

In adults, the liver measures on average $160 \mathrm{~mm}$ in height and $210 \mathrm{~mm}$ in width on a coronal $(\boldsymbol{x z})$ plane [27], [28]. Two inclinations of the insertion direction, on an axial plane $(x y)$ and on a sagittal plane $(z y)$, are required to replicate the manual procedure [7]. The lesions normally treated with LA vary in diameter from $10 \mathrm{~mm}$ to $50 \mathrm{~mm}$ [5]. Consultations with clinicians indicated that a targeting accuracy better than 5 $\mathrm{mm}$, corresponding to the radius of the smallest lesion, would be sufficient for clinical use. This requirement is in line with that of MRI-guided biopsy for tumors of similar size [11].

The system should be MRI-safe or at least MRI-conditional according to ASTM F2503 [29]. Consequently, magnetically induced forces, induced voltage, and heating on metallic or conductive materials should be minimized. Additionally, the system should not produce image artifacts or noticeable reduction in SNR within the imaging region-of-interest (ROI). In Europe, the Medical Device Directive [30] classifies medical devices and states fundamental safety requirements. The Machinery Directive [31] and the Low Voltage Directive [32] specify further safety requirements in order to reduce the hazard deriving from moving parts, conductors, and the hazard related to noise. Emergency stops and means to isolate the system from all energy sources are also required. Moreover, means for locking the actuated parts in place during needle insertions should be provided. Finally, the components of the robot that come in contact with the needle should be sterilizable o disposable.

Growing evidence suggests that the cost of robotic devices can deter hospitals from introducing new technologies into clinical practice [33]-[35]. For this reason the system should not require expensive special tools but should operate with a standard coaxial needle (Somatex, Teltow, Germany). Finally, the system should make use of standard MRI sequences and should only require minimal training for the operator.

\section{SYSTEM DESIGN}

This section describes the design of the 4-DOF needleguiding robot for MRI-guided LA of liver tumors. The robot performs automatic alignment of a needle guide inside the scanner bore and aids manual needle insertion outside the bore. Automatically inserting the coaxial needle inside the bore, as recently proposed in [14], would allow higher accuracy and further time saving, thanks to simultaneous imaging and closed loop control. However, in this case the space inside the scanner does not allow approaching the patient with a standard rigid needle of sufficient length, representing a major technical challenge. Employing a manual insertion of needle and ablation probe instead of automating the process keeps the clinicians in direct control. In turn, this simplifies the approval process for clinical trials since the robot does not come in contact with the patient: the only interaction with the patient occurs during the manual needle insertion. Additionally, the size and the complexity of the system are considerably reduced. For similar reasons, manual needle insertion has been preferred in several prototypes of robots for MRI-guided intervention [7], [10], [11], [17], [18].

The needle-guiding robot builds upon our initial 3 DOF proof-of-concept [25] and consists of a fixed gantry and of a remotely actuated unit carrying a needle guide, which contains

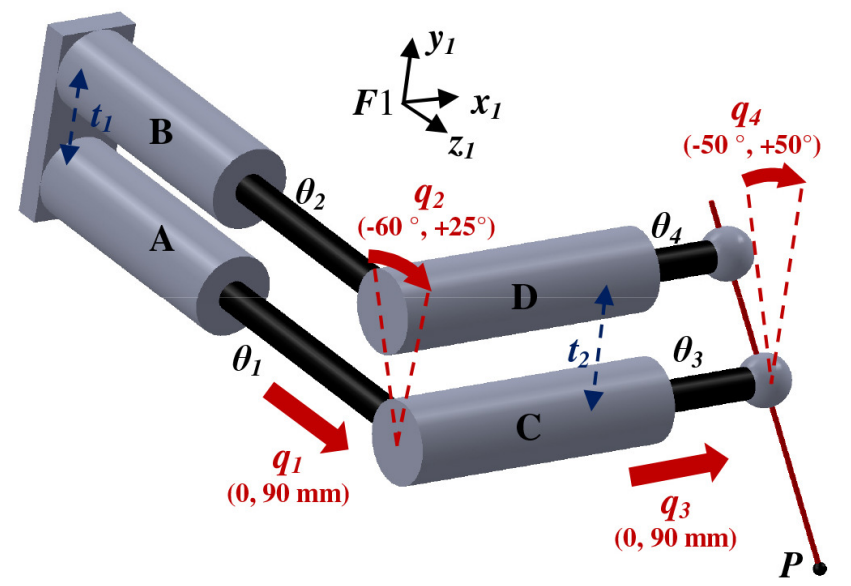

Fig. 3. Kinematic diagram of the needle-guiding robot. Cylinders are A, B, $\mathrm{C}, \mathrm{D}$. The joint coordinates are $q_{1}, q_{2}, q_{3}, q_{4}$. The measured piston positions are $\theta_{1}, \theta_{2}, \theta_{3}, \theta_{4}$. The base frame of the robot is $F 1$. 

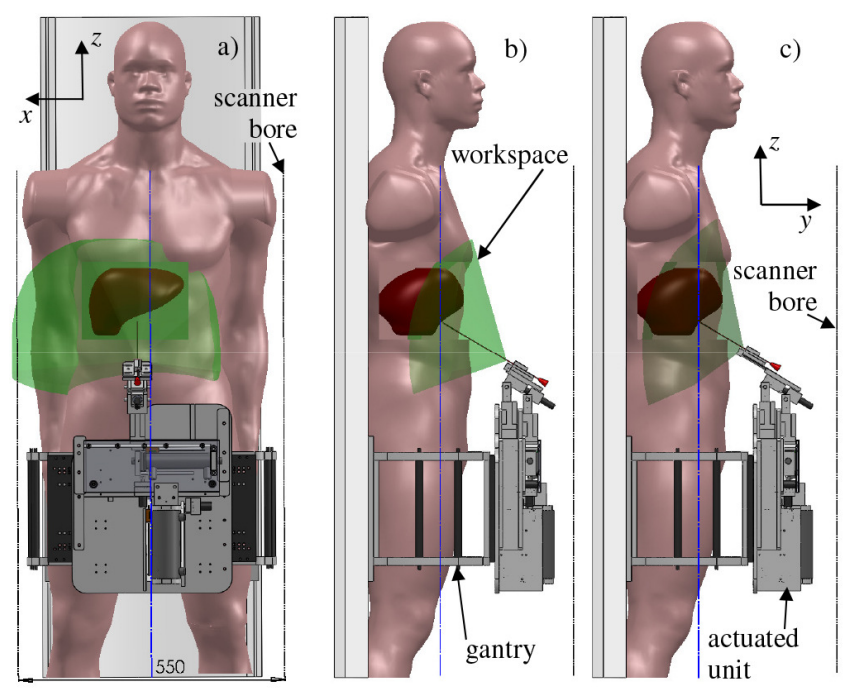

Fig. 4. Workspace of the needle-guiding robot in relation to average liver size in an adult model [28]. Front view (a), side view (b), needle guide extended (c). Dashed lines represent the scanner bore.

one passive marker for registration (Fig. 1). The use of a gantry mounted above the patient is however common to several other robots, such as [18], [36]. The robot is made of plastic materials (Delrin, PTFE) and the needle guide is sterilizable. Additionally, the parts that come in contact with the needle are removable. The passive marker is disposable and it is attached to the non-removable part. The robot is actuated by double-acting plastic cylinders (IPS Inc., USA) supplied by proportional valves (Tecno Basic, Hoerbiger, Germany) that regulate the pressure in the range 0.3 bar to 2.3 bar with an on-board closed loop control and pressure sensor. Pneumatic brakes are employed to lock the cylinders in position during the needle insertion. This safety measure prevents involuntary movements of the needle guide that might pose a risk to the patient. Each brake consists of a miniature pneumatic cylinder with a silicone O-ring that acts as a return spring [25]. The piston position is measured with optical encoders (EM1-250, US Digital). A $9 \mathrm{~m}$ long shielded cable and $9 \mathrm{~m}$ long pipes connect the robot to the control unit, containing valves and control electronics. The control unit includes an emergency stop and is located next to the operator in the MRI control room. Cable and pipes are passed through the waveguides, conventionally installed to connect the control room and the MRI scanner room. In particular, an aluminum

TABLE I

\begin{tabular}{|c|c|c|c|}
\hline & $\begin{array}{l}\text { Liver } \\
\text { model }\end{array}$ & $\begin{array}{c}\text { Workspace } \\
\text { (needle guide } \\
\text { retracted) }\end{array}$ & $\begin{array}{c}\text { Workspace } \\
\text { (needle guide } \\
\text { extended) }\end{array}$ \\
\hline \multicolumn{4}{|l|}{ Front view } \\
\hline \multicolumn{4}{|l|}{ Top view } \\
\hline Volume (1) & 1.80 & 1.05 & 1.69 \\
\hline Coverage $(\%)$ & - & 58 & 94 \\
\hline
\end{tabular}

Simulation results (ref. Fig. 4) refer to the average liver size in adults [28]. wave guide adapter with pi-section filters (5500 $\mathrm{pF}$ Metric Bushing Pi Filter, Tusonix Inc., USA) serves as interface for the shielded cable (XR28F, Maplin, UK) on both sides of the waveguide to prevent EM noise from perturbing the MRI environment [25].

A hybrid serial-parallel structure was chosen for the robot (Fig. 3) since hybrid structures combine larger workspace of serial manipulators with higher rigidity and accuracy of parallel robots. Cylinder A and Cylinder B are mounted in parallel and move the other two along the longitudinal axis of the MRI scanner. They provide translation of the needle guide in $z_{1}$ when moved together $\left(1^{\text {st }} \mathrm{DOF}: q_{1}\right)$ and rotation around $\boldsymbol{x}_{\boldsymbol{1}}$ when moved differentially ( $2^{\text {nd }}$ DOF: $\left.q_{2}\right)$. Similarly, Cylinder $\mathrm{C}$ and Cylinder $\mathrm{D}$ provide translation of the needle guide in $\boldsymbol{x}_{\boldsymbol{1}}$ when moved together $\left(3^{\text {rd }}\right.$ DOF: $q_{3}$ ) and rotation around $z_{1}$ when moved differentially ( $\left.4^{\text {th }} \mathrm{DOF}: q_{4}\right)$. The needle guide is attached to Cylinder $\mathrm{C}$ and Cylinder $\mathrm{D}$ with universal joints. The nominal relation between the joint coordinates $\left(q_{1}, q_{2}, q_{3}\right.$, $\left.q_{4}\right)$ and the measured position of the pistons $\left(\theta_{1}, \theta_{2}, \theta_{3}, \theta_{4}\right)$ is:

$$
\left\{\begin{array}{l}
q_{1}=\theta_{1} \\
q_{2}=\tan ^{-1}\left(\left(\theta_{2}-\theta_{1}\right) / t_{1}\right) \\
q_{3}=\theta_{3} \\
q_{4}=\tan ^{-1}\left(\left(\theta_{4}-\theta_{3}\right) / t_{2}\right)
\end{array}\right.
$$

The parameters $t_{1}$ and $t_{2}$ are the vertical distances between the parallel actuators. The base frame $F 1$ of the robot was assigned with the axes $z_{1}$ and $\boldsymbol{x}_{1}$ parallel to the prismatic joints $q_{1}$ and $q_{3}$ respectively. The needle tip is represented by the point $P$ and the needle length is $l$. The relation between the generic position of $P\left(q_{1}, q_{2}, q_{3}, q_{4}\right)$ in the base frame $F 1$, its initial position $P_{0}(0,0,0,0)$, and the joint coordinates is:

$$
\begin{aligned}
P\left(q_{1}, q_{2}, q_{3}, q_{4}\right)= & T 1\left(q_{1}\right) \cdot T_{2}^{1} \cdot T 2\left(q_{2}\right) \cdot T_{3}^{2} \cdot T 3\left(q_{3}\right) \\
& \cdot T_{4}^{3} \cdot T 4\left(q_{4}\right) \cdot P_{0}
\end{aligned}
$$

The terms $T 1, T 2, T 3, T 4$ are $4 \times 4$ homogeneous transformation matrices, function of the joint coordinates. The misalignment between the joint axes and the coordinate axes, caused by manufacturing and assembly tolerances, is accounted for by the constant matrices $T_{2}^{1}, T_{3}^{2}, T_{4}^{3}$. Independent measurements with an optical tracking system (Optotrak, NDI, Canada, 0.1 $\mathrm{mm}$ nominal accuracy) indicate that the joint axes diverge from the coordinate axes of the base frame $F 1$ by less than 1 degree. Consequently, the matrices $T_{2}^{1}, T_{3}^{2}, T_{4}^{3}$ were treated as identities in this work.

The operator controls the robot based on MRI-guidance and has the following options: 1) align the needle guide to the target point on an axial plane $\left(\boldsymbol{x}_{1} \boldsymbol{y}_{1}\right)$ either with a translation $q_{3}$ or with a rotation $q_{4}$. In either case one joint variable remains unchanged while the other is automatically calculated;2) align the needle guide to the target point on a sagittal plane $\left(z_{1} y_{1}\right)$ either with a translation $q_{1}$ or with a rotation $q_{2} ; 3$ ) input values for any of the joint coordinates to interactively move the robot as required; 4) combine the previous options in any order. While the joints move in a point-to-point fashion, the cylinders follow a constant-jerk trajectory starting at the current position and terminating at the calculated setpoint. 
The linear range of motion of the prismatic joints $q_{1}, q_{3}$ is $90 \mathrm{~mm}$. The angular range of motion of the revolute joints $q_{2}$, $q_{4}$ is $+25^{\circ}$ to $-60^{\circ}$ and $+50^{\circ}$ to $-50^{\circ}$ respectively. In practice, since the needle typically reaches the liver from underneath the rib cage, it is expected that only values of $q_{2}$ within the range $0^{\circ}$ to $-60^{\circ}$ will be of clinical interest. These values were defined in close collaboration with clinicians, considering the large variability in patient size, lesion size and location. Depending on the skill of the clinicians, in conventional manual interventions the needle insertion direction is preferably chosen on an axial plane $\left(q_{2} \approx 0\right)$ to facilitate needle placement [37]. Consequently, providing two inclinations of the needle guide could be particularly beneficial for less experienced operators. Compared to [7], the two rotations of the needle guide are actuated in this robot, since in closed-bore MRI scanners the needle guide cannot be manually inclined by the clinicians. However, our design does not include an actuated translation in the vertical direction due to the limited space above the patient inside the scanner bore. Instead, the needle guide can slide by $50 \mathrm{~mm}$ in order to reach deeper lesions (Fig. 4). The prismatic joints in this robot also have a shorter travel compared to [7] due to the space available in the scanner that restricts the maximum size allowed for the cylinders [25]. The reachable workspace, which consists of the volume defined by the needle tip (insertion depth $\leq 200 \mathrm{~mm}$ ) for all permitted combinations of the joint coordinates, was calculated with simulations. The results indicate that robotassisted needle insertions can target up to $90 \%$ of the liver volume (Table I). As shown in Fig. 4(a), the robot workspace is shifted to the side with respect to the scanner axis in order to match the liver position.

Initially, the robot is secured to the patient bed to prevent any relative motion between the two during the whole procedure. The position of the gantry is chosen based on the location of the lesions known from preoperative MR images. The patient lies under general anesthetic and is closely supervised by the anesthetist throughout the procedure, who also controls the mechanical ventilation. Relative movements between the needle guide and the liver, due to respiration, are minimized by shortly pausing the mechanical ventilation during the same respiratory phase to perform imaging and needle insertion, as in conventional MRI-guided LA procedures [37]. The needle is inserted manually to the depth calculated from the MR images using the depth gauge on the coaxial needle, as in the conventional manual procedure. Typical needle insertion forces are in the range $1 \mathrm{~N}$ to $10 \mathrm{~N}$ [38] and are mostly directed along the needle axis. Additionally, pneumatic brakes are employed to prevent motion of the cylinders during needle insertion (braking force $=20 \mathrm{~N}$ ). A finite element analysis (FEA) indicates that the maximum deflection of the needle guide under a $10 \mathrm{~N}$ transversal load would be smaller than $1 \mathrm{~mm}$ (Fig. 5). The deflection of the coaxial needle (titanium, 14G, Somatex, Teltow, Germany) under the same transversal load applied at mid length would be more than an order of magnitude larger. This suggests that the deflection of the needle guide would be very small compared to needle bending during manual insertions.

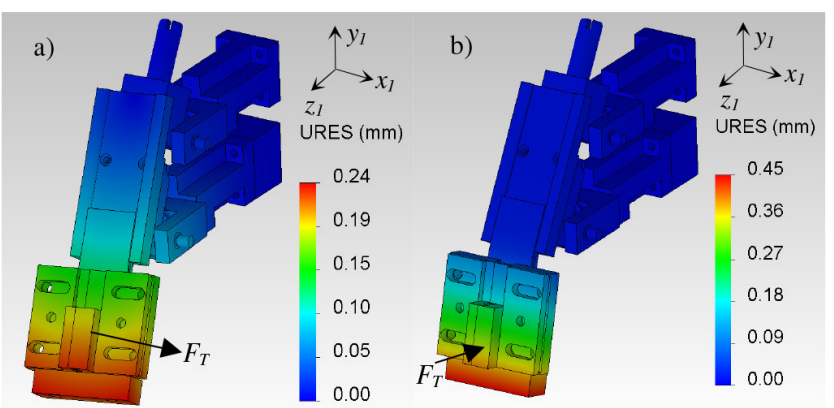

Fig. 5 Deflection of the needle guide under transversal force $F_{T}=10 \mathrm{~N}$.

\section{CONTROLler Design}

This section begins with briefly summarizing the controller requirements for the needle-guiding robot. Subsequently, the model of the pneumatic actuation consisting of a single cylinder is described. The design of a TDC scheme using the backstepping technique is then presented.

\section{A. Controller Requirements}

The needle-guiding robot is intended to carry out point-topoint positioning tasks in a slow and controlled manner. Consequently, the steady-state error should be comparable to the resolution of the position sensor. A low overshoot $(<1$ $\mathrm{mm}$ ) and a short settling time ( $<1$ second) are also desirable. A fast actuation and a high tracking accuracy are not actually required for the needle-guiding robot since the coaxial needle is inserted manually when the pistons are locked in position.

\section{B. System Model}

The pneumatic actuation consists of a double acting cylinder connected through $9 \mathrm{~m}$ long pipes to proportional pressure regulators. The air supply is provided to the proportional valves by the mains in the MRI suite, which is considered an ideal pressure source ( 4 bar nominal). Each proportional valve has an internal pressure sensor and regulates the output pressure according to the control input with an on-board closed loop control. The reaction time quoted by the valve manufacturer is less than $10 \mathrm{~ms}$ and the corresponding bandwidth is above $100 \mathrm{~Hz}$. Consequently, the valve dynamics is much faster than the rest of the system [24]. The pressure dynamics in supply pipes and cylinder chambers can be derived from the state equations of ideal gas assuming isothermal conditions [39]:

$$
\dot{p} \mathcal{V}=Q R T-p \dot{\mathcal{V}}
$$

The terms $p, \mathcal{V}, Q, R, T$ are the absolute gas pressure, the volume of the vessel, the mass flow rate, the gas constant and the gas temperature. The mass flow rate $Q$ through an orifice is calculated from the upstream and downstream pressure [40]:

$$
\left\{\begin{array}{cl}
Q=C p_{i} \rho_{0} \sqrt{T_{0} / T_{i}} \cdot \sqrt{1-\left(\frac{p_{0} / p_{i}-r}{1-r}\right)^{2}} & \frac{p_{0}}{p_{i}}<r \\
Q=C p_{i} \rho_{0} \sqrt{T_{0} / T_{i}} ; & \frac{p_{0}}{p_{i}} \geq r
\end{array}\right.
$$

where $C$ is the conductance of the orifice, $p_{i}$ is the absolute 


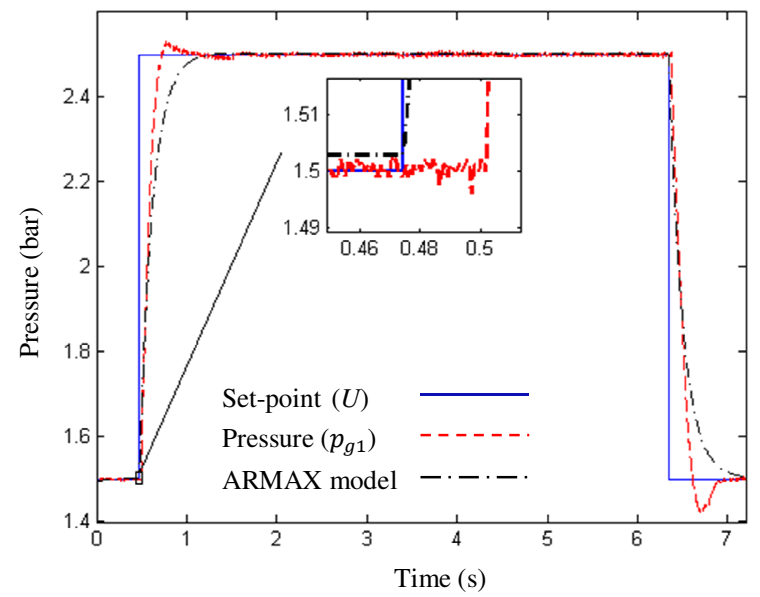

Fig. 6. Pressure measurement at the cylinder's back chamber with $9 \mathrm{~m}$ pipe. Detail view shows the initial delay.

inlet pressure, $\rho_{0}$ is the air density in standard conditions, $T_{0}$ is the reference temperature, $T_{i}$ is the inlet temperature, $p_{0}$ is the reference absolute pressure, and $r$ is the critical pressure ratio corresponding to choked flow. Combining (3) and (4), the pressure in the cylinder chambers can be calculated from the pressure at the valve outlet. In practice, the charge and discharge process might deviate from isothermal [41], while the pressure at the valve outlet depends on the valve dynamics, which is not directly known. As a result, accurate models of the pressure dynamics typically rely on continuous pressure measurements in the cylinder chambers [42]. Consequently, the pressure dynamics was generally disregarded in the design of previous controllers for similar pneumatic systems [20], [21], [24]. In this work, the pressure dynamics due to valve, pipe, and cylinder chamber was experimentally evaluated with a step command issued to the proportional valve and a simultaneous pressure measurement. A pressure sensor (MPX4520GDP, $1 \mathrm{~ms}$ response time, 35 mbar accuracy) and a sampling frequency of $2.5 \mathrm{kHz}$ were used for the experiment in order to achieve a high temporal resolution. The cylinder pressure settles within $2 \%$ of the desired value after 0.5 seconds following the step command and shows an initial delay of $30 \mathrm{~ms}$ (Fig. 6). A discrete-time model of the pressure dynamics was obtained with the ARMAX method [43] in Matlab (Mathworks, Natick, MA). Since in this application the piston is only required to perform slow movements, the pressure dynamics was approximated with a first order transfer function. This choice is based on the following considerations: firstly, the dynamics of the pipe can be described with a first order transfer function as shown in [24]; secondly, the proportional valve has a much higher bandwidth than the rest

TABLE II

SYSTEM PARAMETERS

\begin{tabular}{c|ccccccc}
\hline \hline & $a$ & $a$ & $m$ & $p_{g 2}$ & $F_{a}{ }^{*}$ & $\gamma^{*}$ & $d$ \\
\hline Cylinders A, B & 792 & 508 & 0.3 & 2 & 10 & 20 & - \\
Cylinders C, D & 285 & 158 & 0.15 & 2 & 5 & 20 & - \\
Unit & $\mathrm{mm}^{2}$ & $\mathrm{~mm}^{2}$ & $\mathrm{~kg}$ & bar & $\mathrm{N}$ & $\mathrm{Ns} / \mathrm{m}$ & $\mathrm{N}$ \\
\hline \hline
\end{tabular}

The nominal values of Coulomb friction $F_{a}{ }^{*}$ and viscous friction $\gamma^{*}$ are provided by the cylinder manufacturer. The disturbance $d$ is unknown as it mainly consists of friction forces. of the system. This approach allows simplifying the design and the tuning of the controller, which requires only three parameters, at the cost of lower tracking accuracy. The transfer function corresponding to the ARMAX model in Fig. 6 is:

$$
\frac{p_{g 1}}{U}=\frac{8.6}{s+8.6}
$$

The terms $p_{g 1}$ and $U$ represent the measured pressure relative to atmospheric and the control input of the proportional valve.

The piston dynamics is modelled as in [26]:

$$
a \cdot p_{g 1}-a^{\prime} p_{g 2}-d=m \ddot{x}+\gamma \dot{x}+F_{a} \operatorname{sign}(\dot{x})+F_{m}
$$

The terms $a, a^{\prime}, m$ represent the effective piston areas and the mass of piston and payload. The term $p_{g 1}$ represents the variable pressure in the cylinder back chamber. The term $p_{g 2}$ is the pressure in the cylinder front chamber, which is assumed constant considering the high flow rate provided by the proportional valve and the relatively slow movement of the piston. This assumption was verified with experiments in our previous work [25]. Since $p_{g 1}$ and $p_{g 2}$ are expressed as relative pressures, the contribution of the atmospheric pressure does not appear in (6). The terms $\gamma, F_{a}, F_{m}$ are the viscous, Coulomb friction, and stiction of the cylinder. The term $d$ represents the disturbances that mainly consist of friction forces due to the robot structure. The parameters for both pairs of cylinders are summarized in Table II. The cylinder size was chosen to overcome friction and inertial forces due to the piston and to the structural loads of the robot [26]. The piston position $x$ is measured with the linear encoder while the velocity $\dot{x}$ is calculated by discrete differentiation and low pass filtering. Considering that the unfiltered velocity has low spectral density above $50 \mathrm{~Hz}$, the corner frequency of the filter was set to $120 \mathrm{~Hz}$. This value was chosen since it produces a smooth signal with minimal attenuation in the range of frequency of interest $(0.7 \mathrm{~dB}$ at $50 \mathrm{~Hz})$, while introducing an acceptable delay ( $3 \mathrm{~ms}$ rise time and $5 \mathrm{~ms}$ settling time within $2 \%$ of the final value).

In summary, the complete model of the system, with the approximation adopted for the pressure dynamics, is represented by equations (5) and (6) for the purpose of the controller design.

\section{Backstepping TDC Algorithm}

The control scheme employed is based on TDC formulation [44] which allows estimating disturbances using previous values of the control input and of the state variables. Differently from SMC schemes [24], [25], [42], [45], TDC does not require the assumption of bounded disturbances. This is beneficial here since friction forces caused by the robot structure can be difficult to estimate. Instead, TDC is based on the assumption of bounded variation of the disturbances, which is typically valid if the sampling period is small compared to the system dynamics. The implementation presented here is designed with the backstepping technique [46]. Differently from previous TDC schemes [26], this controller takes into account the pressure dynamics. Differently from fuzzy-logic and neural network controllers 
[47], from SMC schemes [42], [45], and from other TDC schemes [48], the control law does not rely on pressure or force measurements in proximity of the cylinder. This aspect has particular advantages in the MRI environment since commercially available pressure and force sensors are typically not MRI-safe [29].

For the purpose of the controller design, the system model expressed by (5) and (6) is further simplified in accordance with [44] combining the structural loads of the robot and the friction forces in the lumped disturbance $d$ '. The system model for a single cylinder is rewritten as:

$$
\begin{aligned}
& \dot{x_{1}}=x_{2} \\
& \dot{x_{2}}=\left(a x_{3}-d^{\prime}-a^{\prime} p_{g 2}\right) / m \\
& \dot{x_{3}}=8.6\left(U-x_{3}\right)
\end{aligned}
$$

where the sates $x_{1}, x_{2}, x_{3}$ represent the position $x$, the velocity $\dot{x}$ and the pressure $p_{g 1}$. TDC is based on the assumption of bounded variation of the lumped disturbance $d$ ' over the sampling interval $\tau$ :

$$
\left|d^{\prime}(t)-d^{\prime}(t-\tau)\right| \leq \xi
$$

Adopting a small sampling period $\tau$ compared to the system dynamics verifies (8) and the term $d$ ' can be estimated from (7) written for the previous sampling interval:

$$
d^{\prime} \approx a \cdot x_{3}(t-\tau)-a^{\prime} p_{g 2}-m \dot{x_{2}}(t-\tau)
$$

The acceleration at the previous sampling interval is computed by differentiating the piston velocity as in [44]. The inaccuracy in the estimate of $d$ ' resulting from this operation is accounted for within $\xi$ in (8) for simplicity. Since system (7) is in strictfeedback form, the control law is defined using the backstepping method [46] illustrated in the following 3-step procedure. The Steps I and II aim to stabilize the subsystems of (7), while Step III defines the control law.

Step I. Only the first equation in (7) is considered, where $x_{1}$ is the system state and $x_{2}$ is taken as a virtual control. The prescribed position and velocity of the piston are $x_{1 D}$ and $x_{2 D}$. Tracking error $z_{1}$ and Lyapunov function $V_{l}$ are defined as:

$$
\begin{aligned}
& z_{1}=x_{1 D}-x_{1} \\
& V_{1}=\frac{1}{2} z_{1}{ }^{2}>0
\end{aligned}
$$

The virtual control input $x_{2}$ is chosen in order to make the derivative $\dot{V}_{1}$ negative definite. Differentiating (10) and substituting (7) we obtain:

$$
\begin{aligned}
& \dot{z}_{1}=x_{2 D}-x_{2} \\
& \dot{V}_{1}=z_{1} \dot{z}_{1}=-c_{1} z_{1}^{2}<0
\end{aligned}
$$

where $c_{1}>0$ is a design parameter. Smaller values of $c_{1}$ result in weaker control action and slower convergence. The stabilizing function that satisfies (11) is:

$$
\widetilde{x_{2}}=x_{2 D}+c_{1} z_{1}
$$

Step II. The second equation in (7) is now considered: $x_{3}$ is taken as a virtual control, while $x_{2}$ is now a system state. The error $z_{2}$ and the second Lyapunov function $V_{2}$ are introduced:

$$
\begin{aligned}
z_{2} & =\widetilde{x_{2}}-x_{2} \\
V_{2} & =\frac{1}{2} z_{1}{ }^{2}+\frac{1}{2} z_{2}{ }^{2}>0
\end{aligned}
$$

The virtual control $x_{3}$ is chosen to make the derivative $\dot{V}_{2}$ negative definite. Differentiating (13) and substituting (7) and (12) we obtain:

$$
\begin{aligned}
& \dot{z}_{1}=z_{2}-c_{1} z_{1} \\
& \dot{z_{2}}=\dot{x}_{2 D}+c_{1} \dot{z}_{1}-\left(a x_{3}-d^{\prime}-a^{\prime} p_{g 2}\right) / m \\
& \dot{V}_{2}=z_{1} \dot{z}_{1}+z_{2} \dot{z}_{2}=-c_{1} z_{1}{ }^{2}-c_{2} z_{2}{ }^{2}<0
\end{aligned}
$$

The stabilizing function that verifies (14) is:

$$
\widetilde{x_{3}}=\frac{d^{\prime}}{a}+\frac{a^{\prime} p_{g 2}}{a}+\frac{m}{a}\left(\dot{x}_{2 D}+c_{1}\left(z_{2}-c_{1} z_{1}\right)+c_{2} z_{2}+z_{1}\right)
$$

Substituting $d$ ' from (9) and indicating the values at time $(t-\tau)$ with the superscript $\tau$, equation (15) is approximated as:

$$
\widetilde{x_{3}}={\widetilde{x_{3}}}^{\tau}+\frac{m}{a}\left(\dot{x}_{2 D}-{\dot{x_{2}}}^{\tau}+c_{1}\left(z_{2}-c_{1} z_{1}\right)+c_{2} z_{2}+z_{1}\right)
$$

For verification, (16) is substituted back into the Lyapunov function derivative $\dot{V}_{2}$. Under the assumption of bounded variations of the disturbance $d^{\prime}$ (8) we obtain:

$$
\dot{V}_{2} \leq-c_{1} z_{1}{ }^{2}-\left|z_{2}\right|\left(c_{2}\left|z_{2}\right|-\xi / m\right)
$$

Consequently $\dot{V}_{2}<0$ for $\left|z_{2}\right|>\xi / m c_{2}$ hence the sub-system consisting of the first two equations in (7) is bounded.

Step III. The third equation in (7) is now considered, where $x_{3}$ is a system state and $U$ is the actual control input. The error $z_{3}$ and the third Lyapunov function $V_{3}$ are defined as:

$$
\begin{gathered}
z_{3}=\widetilde{x_{3}}-x_{3} \\
V_{3}=\frac{1}{2} z_{1}{ }^{2}+\frac{1}{2} z_{2}{ }^{2}+\frac{1}{2} z_{3}{ }^{2}>0
\end{gathered}
$$

Differentiating (18) and substituting (7) and (16) we obtain the following expressions that contain directly the control input $U$ :

$$
\begin{gathered}
\dot{z_{2}}=\frac{a}{m} z_{3}-c_{2} z_{2}-z_{1}-\frac{1}{m}\left(d^{\prime \tau}-d^{\prime}\right) \\
\dot{z_{3}}=\frac{m}{a}\left(\ddot{x}_{2 D}+c_{1}\left(\dot{z_{2}}-c_{1} \dot{z_{1}}\right)+c_{2} \dot{z_{2}}+\dot{z_{1}}\right)-8.6\left(U-x_{3}\right) \\
\dot{V}_{3}=z_{1} \dot{z}_{1}+z_{2} \dot{z_{2}}+z_{3} \dot{z_{3}}=-c_{1} z_{1}{ }^{2}-c_{2} z_{2}{ }^{2}-c_{3} z_{3}{ }^{2}<0
\end{gathered}
$$

The control law resulting from the previous analysis is:

$$
\begin{aligned}
U=\frac{m}{8.6 a}\left(\ddot{x}_{2 D}+\right. & \left(z_{2}-c_{1} z_{1}\right)\left(1-c_{1}{ }^{2}\right) \\
& \left.+\left(c_{2}+c_{1}\right)\left(\frac{a}{m} z_{3}-z_{1}-c_{2} z_{2}\right)\right) \\
& +\left(\frac{a}{m} z_{2}+c_{3} z_{3}\right) \frac{1}{8.6}+x_{3}
\end{aligned}
$$

The proportional valve regulates its output pressure according to the control input $U$ with the on-board control loop. The term $x_{3}$ is estimated from the control input at the previous sampling interval using the model of the pressure dynamics (5). This approximation introduces an error $\varepsilon$ in the Lyapunov derivative $\dot{V}_{3}$ that consequently becomes: 


$$
\begin{aligned}
\dot{V}_{3} \leq-c_{1} z_{1}{ }^{2}- & \left|z_{2}\right|\left(c_{2}\left|z_{2}\right|-\xi / m\right) \\
& -\left|z_{3}\right|\left(c_{3}\left|z_{3}\right|-8.6 \varepsilon-\left(c_{1}+c_{2}\right) \xi / a\right)
\end{aligned}
$$

If $\left|z_{3}\right|>\left(8.6 \varepsilon / c_{3}+\left(c_{1}+c_{2}\right) \xi / a c_{3}\right)$ and $\left|z_{2}\right|>\xi / m c_{2}$ then $\dot{V}_{3}<0$ and system (7) is bounded with the control law (20). As a result, $V_{3}$ and $z_{1}, z_{2}, z_{3}$ converge to arbitrarily small values depending on the tuning parameters $c_{1}, c_{2}, c_{3}$.

\section{ROBOT REGISTRATION}

The targeting accuracy of the needle-guiding robot depends not only on the control algorithm but also on registration, which relates the coordinate frame $F 1$ of the robot to the frame F0 of the MRI scanner. Similarly to [25], a single marker is mounted on the needle guide (Fig. 1), which is translated twice in the orthogonal directions $z_{1}$ and $\boldsymbol{x}_{1}$, while in each position the marker is localized based on the MR images. This procedure defines 3 points which uniquely identify the transformation between $F 1$ and $F 0$. Since the marker is mounted on the needle guide, the registration is only minimally affected by the tolerances of the robot structure. Using a single maker and moving the needle guide has the following advantages: firstly, the needle guide is compact; secondly, the distance between the points used for registration depends on the translation of the needle guide and can therefore be large, resulting in smaller angular errors. A sufficient separation between the points is $40 \mathrm{~mm}$, as indicated by the simulations studies of the marker localization method. While this approach requires three sets of MR images, the scan time can be reduced by appropriately selecting the sequence parameters based on the known robot movements.

The marker was localized with an image processing method based on the convolution theorem of Fourier transforms [49]. Differently from our previous work that made use of semiactive markers [25], a passive marker is employed here (.43 Caliber Clear Paintballs 8000, Rap4 UK). While fast and accurate tracking of semi-active markers was demonstrated in [50], a closer integration with the scanner was required. The main advantage of the approach presented here is its portability, since it does not rely on special imaging sequences, which would have to be reprogrammed for different MRI scanners. This aspect is particularly beneficial for this system, which is employed on different scanners (Siemens, Verio; GE, Discovery MR750). Additionally, passive markers do not experience heating as a consequence of electromagnetic coupling [51] and are therefore completely safe. The main drawback is the time associated to the processing of multiple slices that precludes the use of this method for real time tracking of the marker. Since the needleguiding robot can be tracked using the encoder measurements and since liver motion during needle insertion is minimized by shortly pausing the mechanical ventilation [37], this limitation is considered acceptable. Passive markers produce lower intensity signals compared to their semi-active counterparts and are more sensitive to noise generated by neighboring objects. In this case, this problem is solved by mounting the marker on the needle guide, which remains always above the volume occupied by the patient in the MRI scanner.

The localization method relies on the spherical shape of the marker. Similarly to [52], the Fourier Filtering Method [53] is employed. However, it is applied here to a volume consisting of adjacent slices instead of individual 2D images. As a result, the marker's position can be computed at any instant from a predefined set of slices, without simultaneously updating the sequence parameters on the MRI scanner. Instead, in [52] the position of two slices containing the marker should be constantly updated, which required the use of special imaging sequences. From [53], the relationship between the Fourier transforms $M$ and $I$ of the acquired volume and of a template sphere of known radius is:

$$
M=I \times \Delta+N
$$

where $N$ is the noise and $\Delta$ is the Fourier transform of the delta functions that identify the position of the sphere. A Wiener filter is used to reduce the noise produced by other objects. Consequently, $N$ is neglected in (22) and the sphere position $\left(x_{0}, y_{0}, z_{0}\right)$ is calculated using the inverse Fourier transform:

$$
\delta\left(x_{0}, y_{0}, z_{0}\right)=F F T^{-1}(M / I)
$$

The division of the Fourier transformed images $M$ and $I$ is computed dividing the magnitudes and subtracting the phase values. The voxels in the region with highest intensity correspond to the template sphere. The region's center of mass weighted by intensity is then calculated achieving sub-voxel accuracy. Finally, a confirmation image is displayed to verify that the feature corresponds to the marker. Simulations indicate that the accuracy of the localization method is better than 0.3 voxels. Consequently, a mutual distance between the points larger than $30 \mathrm{~mm}$ would result in angular errors smaller than 1 degree. Targeting experiments in the MRI scanner demonstrated errors of $1.5 \mathrm{~mm} \pm 1.1 \mathrm{~mm}$ for the localization method [52]. Similar results are expected for this implementation, since it relies on the same formulation, and will be verified experimentally as part of our future work.

\section{EXPERIMENTAL RESULTS}

Experimental tests have been conducted to evaluate the MRI-compatibility of the system, the performance of the controller, and the targeting accuracy of the needle-guiding robot in the MRI scanner.

\section{A. MRI-Compatibility}

The MRI-compatibility of the system was evaluated with tests in a 3 T closed-bore MRI scanner (Siemens, Verio). The system was set up as follows: 1) control unit located in the MRI control room; 2) needle-guiding robot positioned on the scanner bed above a cylindrical phantom (Nickel Sulphate solution) aligned with the scanner isocenter (Fig. 7); 3) air supply lines and encoder cable connected to the cylinders and to the control unit. In particular, the phantom was placed centrally on an axial slice (Fig. 7(a)) since a centered regionof-interest was considered for the subsequent analysis, as specified by the relevant standards [54], [55]. The needleguiding robot was set up in the home position $\left(q_{1}, q_{2}, q_{3}, q_{4}=0\right)$. 


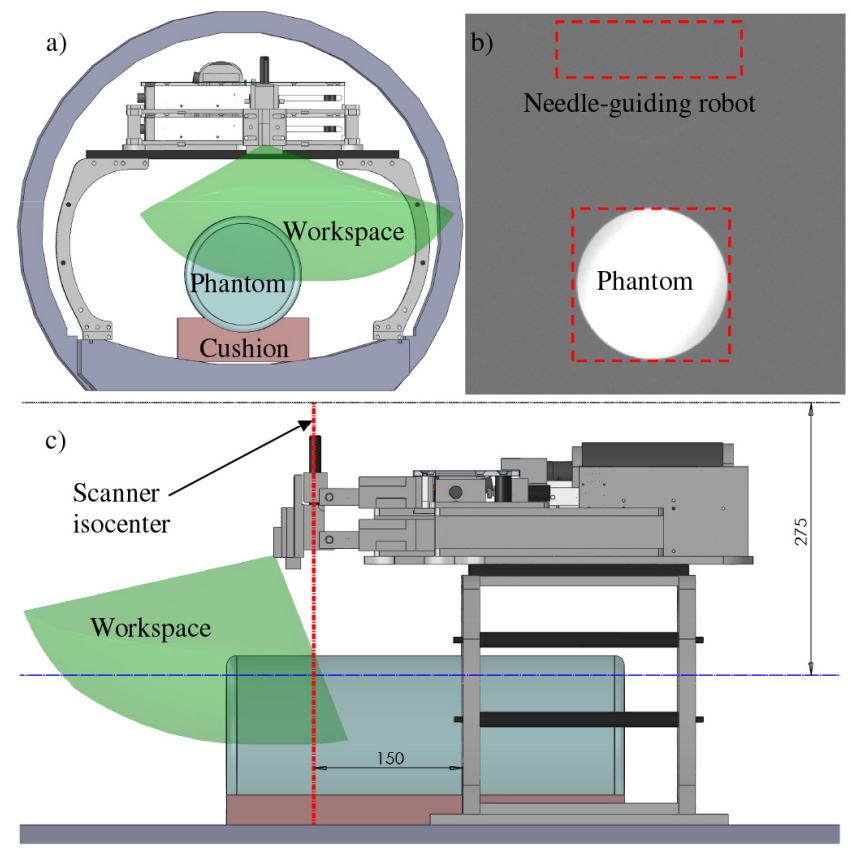

Fig. 7. CAD model of the test set-up in a $3 T$ MRI scanner (a); axial MR image (TrueFISP) representative of the robot powered on (b); lateral view of the set-up (c). The robot is in the home position $\left(q_{1}, q_{2}, q_{3}, q_{4}=0\right)$. The coaxial needle is not part of the set-up since it is known to create image artifacts.

The effects of the system on the MR images were assessed considering visible image artifacts, variations in SNR, and image uniformity. The imaging sequences used were a True Fast Imaging with Steady State Precession (TrueFISP, TR = $4.4 \mathrm{~ms}, \mathrm{TE}=2.2 \mathrm{~ms}, \mathrm{FA}=44^{\circ}, \mathrm{FOV}=400 \times 400 \mathrm{~mm}$, Slice Thickness $=8 \mathrm{~mm}$ ) and a Turbo Spin Echo $($ TSE, TR $=3000$ $\mathrm{ms}, \mathrm{TE}=12 \mathrm{~ms}, \mathrm{FA}=180^{\circ}, \mathrm{FOV}=300 \times 300 \mathrm{~mm}$, Slice Thickness $=8 \mathrm{~mm}$ ) with the scanner Body Coil. Initially, the phantom was imaged alone to define a baseline condition. Subsequently, the needle-guiding robot was positioned in the scanner and MR images were acquired, first with the control unit disconnected from the robot, then with the robot activated but not moving, and finally with all pistons in motion. The MR images did not show visible artifacts in any of the conditions tested (Fig. 8) while the variation in SNR calculated according to [54] remained below 5\% in all test conditions (Table III). While negative variations correspond to loss in SNR $(<1 \%)$, positive variations are usually attributed to experimental measurement errors and do not indicate image degradation [19]. These results are comparable to our previous tests [25]

\begin{tabular}{c|cccc}
\multicolumn{4}{c}{ TABLE III } \\
MRI SNR STUDY \\
\hline \multirow{2}{*}{ Phantom } & \multicolumn{2}{c}{ TSE } & \multicolumn{2}{c}{ TrueFISP } \\
& SNR & Variation & SNR & Variation \\
Power off & 411 & - & 124.7 & - \\
Power on & 410 & $-0.24 \%$ & 129.6 & $3.96 \%$ \\
Movement & 410 & $1.70 \%$ & 126.6 & $1.56 \%$ \\
\hline \hline
\end{tabular}

Test in 3T MRI scanner: SNR calculated according to [54].

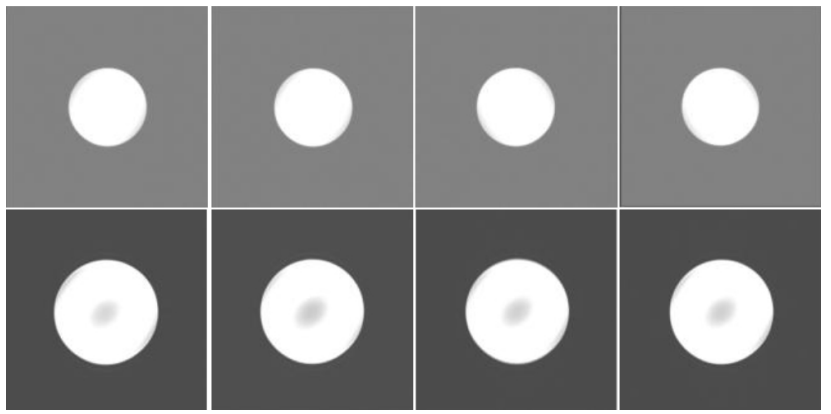

Fig. 8. MR images. From left to right: Phantom, Power off, Power on, Movement. Top row refers to TrueFISP; bottom row refers to TSE.

TABLE IV

MR IMAGE UNIFORMITY STUDY

\begin{tabular}{c|cccc}
\hline \hline & \multicolumn{2}{|c}{ TSE } & \multicolumn{2}{c}{ TrueFISP } \\
& PDNU \% & NAADU \% & PDNU \% & NAADU \% \\
\hline Phantom & 29.9 & 89.1 & 47.9 & 77.8 \\
Power off & 35.4 & 86.8 & 48.4 & 77.9 \\
Power on & 31.7 & 88.5 & 49.2 & 77.9 \\
Movement & 31.9 & 88.4 & 48.9 & 77.9 \\
\hline \hline
\end{tabular}

Test in 3T MRI scanner: PDNU and NAADU calculated according to [55].

and confirm that the system does not have noticeable effects on the MR images.

As recently proposed in [13], image uniformity was assessed alongside SNR in order to evaluate the effect of the needle-guiding robot in the region-of-interest of the MR images. The peak deviation nonuniformity (PDNU) and the normalized absolute average deviation uniformity (NAADU), as defined in [55], were computed for both imaging sequences (Table IV). Differently form [13], no prefiltering was employed on the images. The results show a limited variation of both indexes due to the presence of the robot. For the TSE sequence, the largest variation compared to the baseline is observed with the robot in the power-off condition. Smaller variations correspond to the configurations power-on and movement, which are the most relevant for the clinical use. This effect is different depending on the imaging sequences and will be further investigated as part of our future work. No effect on the encoder reading was detected as a result of the MRI environment and of the waveguide adapter in either static or dynamic tests. In practice, interleaved imaging and motion is employed to avoid motion artifacts on the MR images [11].

\section{B. Controller Performance}

The analysis presented in this section is intended to highlight the differences between the TDC scheme (20) and an SMC algorithm designed for the same system [25]. The comparative study specifically refers to pneumatic actuations with long supply lines, defined as in previous works [20], [24]. The performance of the controllers was evaluated for a single cylinder mounted horizontally, similarly to the actuators in the robot (Fig. 9), since this approach is more general and easily reproducible [20], [24]. The SMC algorithm was presented in detail in [25] and will only be briefly summarized here: 


$$
\left\{\begin{array}{c}
U=u_{e}+u_{1}+u_{2} \\
u_{e}=\frac{a^{\prime} p_{g 2}}{a}+\frac{\dot{x} \gamma^{*}}{a}+\frac{m \ddot{x}_{D}}{a}+\frac{m}{a}\left(\dot{x_{D}}-\dot{x}\right) \lambda+\frac{F_{a}^{*}}{a} \operatorname{sign}(\dot{x}) \\
u_{1}=\left(\frac{\Psi}{a}+\frac{\Gamma}{a}|\dot{x}|+\eta\right) \cdot \operatorname{sat}\left(\frac{\lambda\left(x_{D}-x\right)+\left(x_{D}-\dot{x}\right)}{b}\right) \\
u_{2}=\left(\frac{\Psi}{a}+\frac{\Gamma}{a}|\dot{x}|\right) \cdot \operatorname{sat}\left(\frac{\lambda\left(x_{D}-x\right)+\left(x_{D}-\dot{x}\right)}{g}\right)
\end{array}\right.
$$

The terms $\gamma^{*}$ and $F_{a}{ }^{*}$ are the nominal values of viscous and Coulomb friction of the piston (ref. Section IV). The terms $\Gamma$ and $\Psi$ are the maximum variations of viscous and Coulomb friction which are assumed equal to $\gamma^{*}$ and $F_{a}^{*}$ respectively. The parameters $b$ and $g$ are the slope of the saturation function sat $(\bullet)$, while $\eta$ and $\lambda$ are design parameters affecting responsiveness and damping. The pressure dynamics due to valve and pipe was not considered in this control scheme.

The algorithms were programmed on a microcontroller (mbed NXP LPC1768) at $1.5 \mathrm{kHz}$ sampling frequency. The controller parameters for both schemes were manually tuned for low steady state error, low overshoot, and short settling time (Table V). In order to evaluate the robustness of the controllers to model uncertainties and disturbances, tests were also conducted with an additional $2.5 \mathrm{~kg}$ payload, which exceeds the mass of the moving parts in the needle-guiding robot. As observed in [20], [26], [42], [45], that employ a similar approach, the payload also results in additional friction forces. Notably, the cylinders employed in this system have a

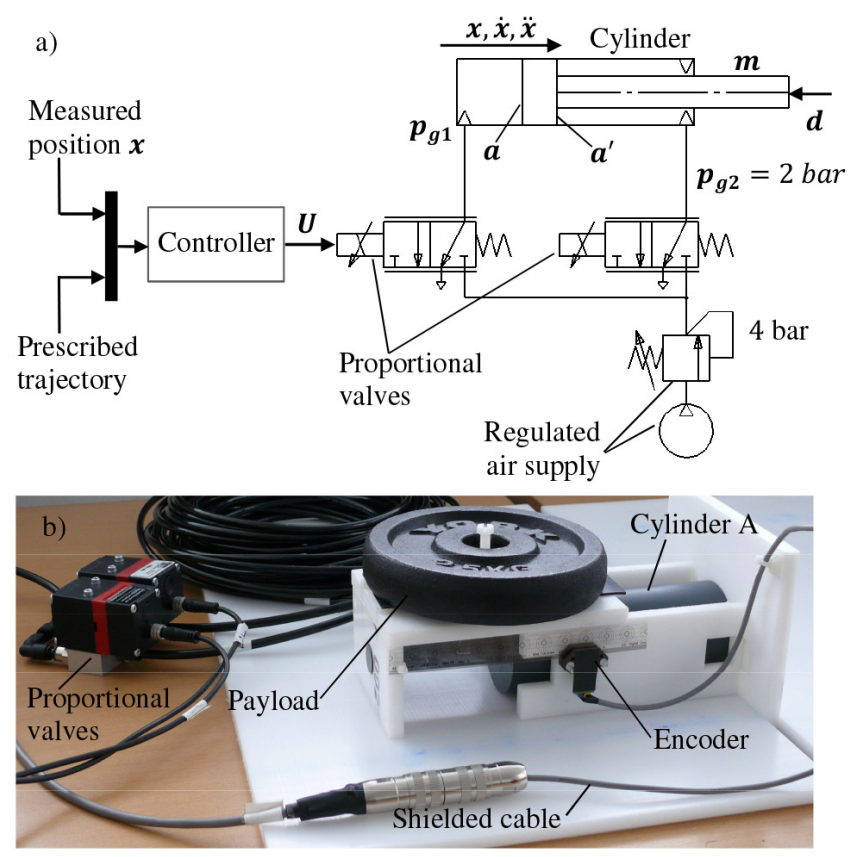

Fig. 9 Pneumatic actuation: (a) schematic of the pneumatic circuit; (b) test set-up, representative of the individual cylinders in the needle-guiding robot.

TABLE V

CONTROLLER PARAMETERS FOR CYLINDER A

\begin{tabular}{c|cccc|ccc}
\multicolumn{8}{c|}{ CONTROLLER PARAMETERS FOR CYLINDER A } \\
\hline \hline Controller & \multicolumn{3}{|c|}{ SMC scheme (24) } & \multicolumn{2}{|c}{ TDC scheme (20) } \\
\hline Symbol & $\lambda$ & $b$ & $g$ & $\eta$ & $c_{1}$ & $c_{2}$ & $c_{3}$ \\
Value & 10 & 100 & 4 & 0.75 & 5 & 30 & 5 \\
Unit & $\mathrm{Hz}$ & $\mathrm{mm} / \mathrm{s}$ & $\mathrm{mm} / \mathrm{s}$ & $\mathrm{bar}$ & $\mathrm{Hz}$ & $\mathrm{Hz}$ & $\mathrm{Hz}$ \\
\hline \hline
\end{tabular}
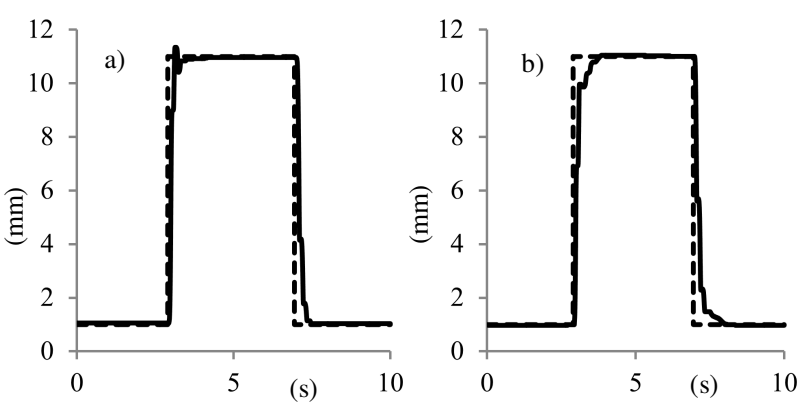

Fig. 10. Step response for Cylinder A without payload: (a) SMC Scheme (24), (b) TDC Scheme (20). Results are similar for other test conditions.

TABLE VI

STEP RESPONSE FOR CYLINDER A

\begin{tabular}{c|cc|cc}
\hline \hline Controller & \multicolumn{2}{|c|}{ SMC (24) } & \multicolumn{2}{c}{ TDC (20) } \\
\hline $\begin{array}{c}\text { Load (kg) } \\
\begin{array}{c}\text { Overshoot } \\
(\mathrm{mm})\end{array}\end{array}$ & 0 & 2.5 & 0 & 2.5 \\
$\begin{array}{c}\text { Settling } \\
\text { time (s) }\end{array}$ & 0.45 & 0.51 & 0.68 & 0.78 \\
$\begin{array}{c}\text { Steady-state } \\
\text { error (mm) }\end{array}$ & 0.12 & 0.16 & 0.02 & 0.03 \\
\hline \hline
\end{tabular}

$\overline{\text { Values are the maximum over a set of } 5 \text { steps with amplitudes } 0.5}$ $\mathrm{mm}, 1 \mathrm{~mm}, 5 \mathrm{~mm}, 10 \mathrm{~mm}$, and $20 \mathrm{~mm}$ in both directions of motion. Settling time refers to $5 \%$ of the step amplitude.

larger friction (Table II) compared to the moving parts of the robot, which were manufactured from low-friction materials (the coefficients of friction for Delrin and PTFE are respectively 0.2 to 0.35 and 0.08 to 0.14 , DuPont Inc.).

The controllers were tested with a series of step responses of different amplitude $(0.5 \mathrm{~mm}, 1 \mathrm{~mm}, 5 \mathrm{~mm}, 10 \mathrm{~mm}, 20 \mathrm{~mm})$. The step response for one test condition is depicted in Fig. 10. The maximum values of steady-state error, overshoot, and settling time for each controller are reported in Table VI. Notably, both controllers meet the requirements of overshoot and settling time defined in section IV.A, which indicates that the tuning adopted is in fact appropriate for the application. The SMC scheme (24) achieved a shorter settling time than TDC (20). This is due to the lack of integral action in this controller which conversely limits the position accuracy if the system parameters and the disturbances are not exactly known. The TDC scheme achieved smaller overshoot and steady-state error but longer settling time. Although the SMC scheme should in theory not produce overshot, in practice this is only the case if the system parameters are accurately known. Overshoot and oscillations were in fact observed experimentally with SMC schemes in [24] and [45].

The tracking performance was evaluated with a periodic constant-jerk polynomial trajectory (Fig. 11). The SMC scheme achieved smaller root-mean-square error (RMSE) and smaller tracking error (Table VII) due to its higher responsiveness but its performance degraded more with payload. In both cases the largest errors correspond to the points with maximum velocity in the reference trajectory, suggesting that the long supply lines are limiting the responsivenes of the system regradless of the controller used. The control input for the tracking test is depicted in Fig. 12. 

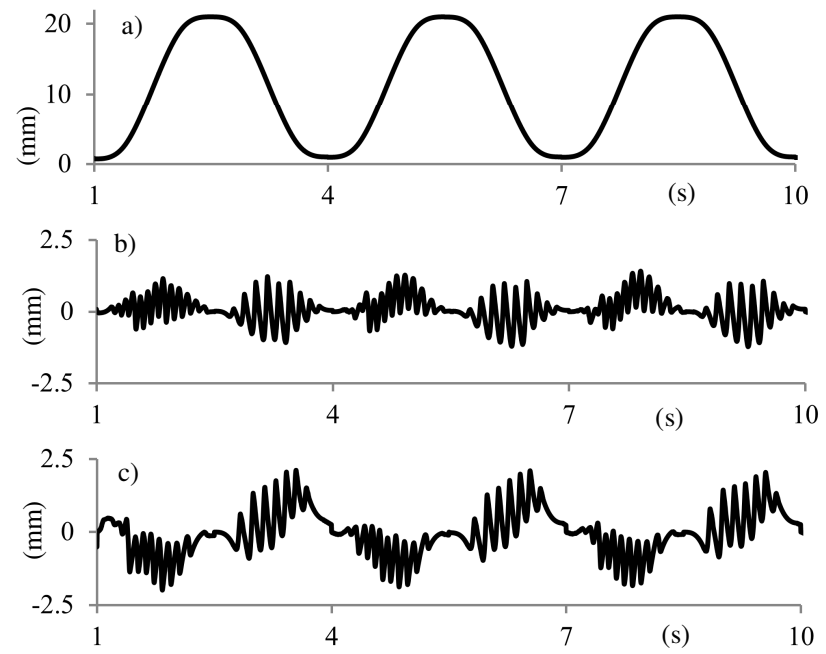

Fig. 11. Tracking error for Cylinder A without payload: (a) polynomial constant-jerk trajectory; (b) SMC Scheme (24); (c) TDC Scheme (20).

For both algorithms the controlled pressure was limited in the range 0.3 bar to 2.3 bar (ref. Section III). However, the size of the pneumatic cylinder was chosen according to these limits, while also including a safety factor [26]. The SMC scheme shows smaller variations of the control action, due to the bounded amplitude of the saturation functions in (24), which follows from the assumption of bounded disturbances. If this assumption is not met, the performance of the SMC scheme degrades, as shown by the tests with payload. Conversely, the TDC scheme results in higher variations of the control action and proves more robust to model uncertainties. As shown in Fig. 12, the TDC scheme reaches the pressure limits more frequently than the SMC: while lifting these limits would result in a more responsive control with both schemes, the TDC algorithm is likely to show the largest improvement.

The SMC and the TDC algorithms are similarly simple to program on the microcontroller and both rely only on position measurements. The SMC scheme (24) requires tuning four parameters $(\lambda, b, g, \eta)$. Additionally, assumptions should be made on the magnitude of the disturbances, which might be difficult to estimate with sufficient accuracy. The TDC algorithm (20) requires tuning three parameters $\left(c_{1}, c_{2}, c_{3}\right)$ and is based on the assumption of bounded variations of the disturbances, which is typically valid at high sampling frequencies. Considering that for this application steady-state accuracy is valued more than tracking accuracy, the TDC scheme (20) appears preferable to the SMC algorithm (24).

\section{MRI-Guided Targeting}

A phantom study was conducted to evaluate the targeting accuracy of the needle-guiding robot in the MRI scanner. The gantry was secured to the patient bed and the system was set up as described in Part A. A porcine gelatin phantom containing four targets made of cod-liver-oil capsules (Boots, UK) and Blu-tack (Bostik, France) was aligned with the scanner isocenter. The size of the phantom (210 mm long, 140 $\mathrm{mm}$ high, $60 \mathrm{~mm}$ deep) is similar to the average size of the liver in adults [27], [28]. In particular, porcine gelatin is

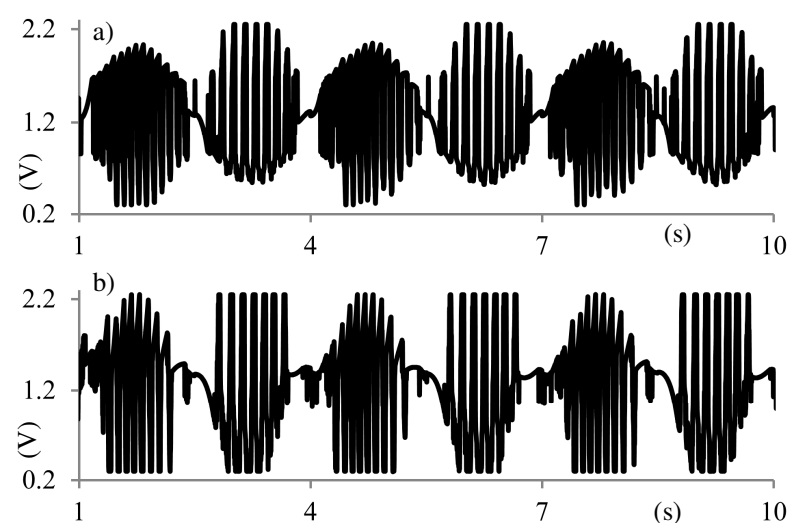

Fig. 12. Control input for Cylinder A without payload: (a) SMC Scheme (24); (b) TDC Scheme (20).

TABLE VII

TRACKING ERROR FOR CYLINDER A

\begin{tabular}{c|cc|cc}
\hline \hline Controller & \multicolumn{2}{|c|}{ SMC (24) } & \multicolumn{2}{c}{ TDC (20) } \\
\hline Load (kg) & 0 & 2.5 & 0 & 2.5 \\
Maximum & 1.49 & 1.78 & 2.12 & 2.24 \\
error (mm) & 0.47 & 0.60 & 0.74 & 0.75 \\
RMSE (mm) & 0.45 & & & \\
\hline \hline
\end{tabular}

Reference trajectory: $20 \mathrm{~mm}$ peak-to-peak amplitude and $3 \mathrm{~s}$ period.

commonly employed in needle insertion experiments since it is representative of biological tissues. The targets are oval with diameters between $10 \mathrm{~mm}$ and $15 \mathrm{~mm}$, which is representative of the smallest lesions normally treated with LA [5]. Finally, the same needle employed in conventional LA procedures was used (14G, diamond tip, Somatex, Germany).

The workflow of the test is represented in Fig. 13. The initial setup included: 1) positioning and securing the robot on the patient bed; 2) running the pipes and the cable to the control unit; 3) connecting the microcontroller to the PC hosting the user interface; 4) verifying the correct operation of the system. The next phase is registration: three sets of MR images $\left(\mathrm{T} 1 \mathrm{fl} 2 \mathrm{D}, \mathrm{TR}=140 \mathrm{~ms}, \mathrm{TE}=2.46 \mathrm{~ms}, \mathrm{FA}=65^{\circ}, \mathrm{FOV}\right.$ $=400 \times 300 \mathrm{~mm}$, Slice Thickness $=3 \mathrm{~mm}$, Slice number $=50$ ) were employed to localize the passive marker mounted on the needle guide (ref. Section V). For simplicity, the same imaging sequence was used for the rest of the experiment.

A graphical user interface (GUI) programmed in Matlab (Mathworks, Natick, MA, USA) was employed to display the MR images and the insertion direction based on the robot position. The target point was selected on the MR images and the needle guide was aligned with the target on two orthogonal planes, moving the actuated joints according to the operator's input (ref. Section III). Based on the piston position, the insertion direction and the insertion depth were calculated from (1), (2) and displayed on the MR images for verification. At this point the position of the marker was measured again from a new set of images for verification. Subsequently, the patient-table was moved out of the scanner bore and the coaxial needle was inserted manually using the needle guide. Finally, a set of verification images was acquired (Fig. 14(a)) and the targeting error was calculated using 3D Slicer [56]. 


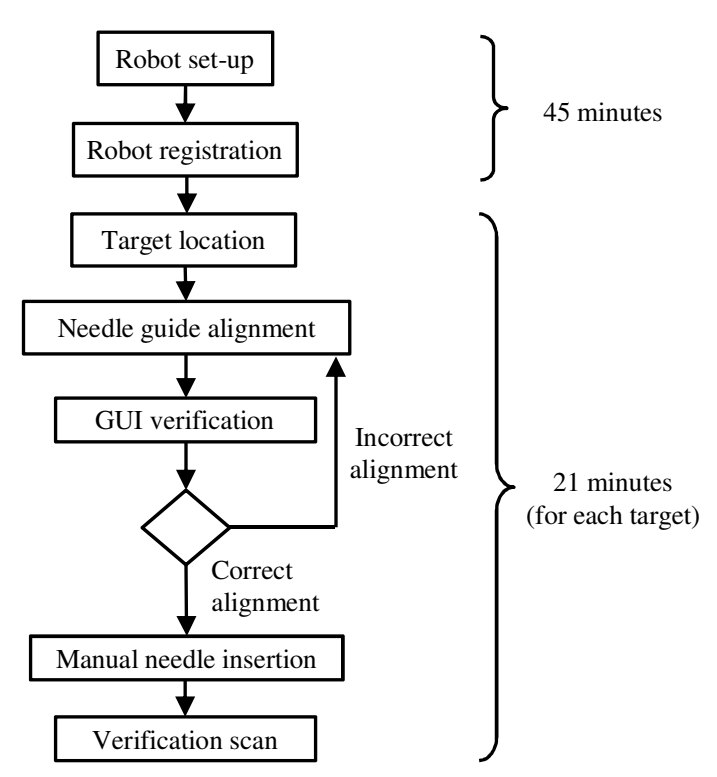

Fig. 13. Workflow of MRI-guided targeting accuracy test.

A total of 10 insertions were performed for 4 different targets (Fig. 14(b)) starting with different values of the joint coordinates and selecting different insertion directions. In particular, the initial starting point corresponded to the home position $\left(q_{1}, q_{2}, q_{3}, q_{4}=0\right)$, while each successive insertion was the starting point for the next. The insertion direction is defined by the joint coordinates $q_{2}, q_{4}$ (ref. Fig. 3). The inplane error reported in Table VIII was calculated as the distance between the center of the target and the center of the needle artifact on the axial slice $(\boldsymbol{x y})$ containing the target [11]. This approach implies two assumptions: firstly, the needle axis is considered at the center of the artifact, as in conventional MRI-guided LA procedures; secondly, the needle tip is considered to be on the same slice as the target, disregarding any errors in insertion depth which are mostly due to the operator. The mean in-plane error is $2.9 \mathrm{~mm}$, with a standard deviation of $1.4 \mathrm{~mm}$ and a maximum of $4.8 \mathrm{~mm}$, which is within the requirements set in Section II. Overall, the results are comparable to those reported in [11] for a similar experiment involving robot-assisted needle insertions (in-plane errors between $1.1 \mathrm{~mm}$ and $3.7 \mathrm{~mm}$ over 7 insertions).

Possible causes of error are: 1) needle bending; 2) deformation of the gelatin phantom; 3) robot tolerances; 4) deflection of the needle guide; 5) marker localization errors; 6) resolution of the MR images; 7) shift of needle artifact in the direction of the frequency encoding gradient. In particular, the error contribution of the robot tolerances was assessed through measurements with an optical tracking system (Optotrak, NDI, Canada). The results indicate that the robot repeatability on the needle guide is better than $1.5 \mathrm{~mm}$. Error contributions of the same magnitude are expected from the marker localization (ref. Section V), while artifact shifts of $0.5 \mathrm{~mm}$ are reported in [11]. Finally, the error contribution of needle guide deflection is expected to be very small compared to needle bending (ref. Section III). A detailed analysis of the different error contributions and of the error variation within the workspace is beyond the scope of this paper and will be conducted as part of our future work. Although the in-plane error does not account for errors in insertion depth, this factor could also affect the targeting accuracy, due to the low resolution of the depth gauge on the coaxial needle $(10 \mathrm{~mm})$. In preparation for the clinical trials, an additional depth gauge will be integrated with the needle guide in order to measure the insertion depth with higher resolution in an attempt to reduce operator error.

The duration of the different phases of the test is reported in Fig. 13. The initial set-up required approximately 30 minutes and was the most time-consuming part; however this could be carried out either before or during the preparation phase in MRI-guided LA (ref. Fig. 2). Robot registration required 15 minutes, mainly consisting of scan time. This phase would be additional to the standard workflow of LA procedures, however its duration could be reduced using faster imaging sequences [57]. Planning and needle insertion were completed on average in 21 minutes, compared to the average 60 minutes of the manual procedure. This is mainly due to the ability offered by the needle-guiding robot to verify the insertion direction prior to the insertion itself, which avoids having to repeatedly move the patient bed out of the bore. As a result, all targets were punctured at the first attempt and there was no need to reinsert the needle. Minimizing the amount of trialand-error in the needle insertion could also enhance safety, since the chance of mistakenly puncturing blood vessels or vital organs would be reduced. The phantom study suggests that a time saving of over 30 minutes for each lesion could be associated with the use of the needle-guiding robot in MRIguided LA procedures. In practice, since HCC has a multifocal origin [1], successive needle insertions can be required to treat different lesions. In such cases, planning and needle insertion, which represent the most time consuming part of the procedure (ref. Fig 2), need to be repeated for each target. Therefore the time saving associated with the robot-assisted needle insertion could be even bigger.

TABLE VIII

TARGETING ERROR

\begin{tabular}{c|cccccccccc}
\hline \hline $\begin{array}{c}\text { Insertion } \\
\text { number }\end{array}$ & 1 & 2 & 3 & 4 & 5 & 6 & 7 & 8 & 9 & 10 \\
\hline Target & $\mathrm{D}$ & $\mathrm{D}$ & $\mathrm{B}$ & $\mathrm{B}$ & $\mathrm{A}$ & $\mathrm{C}$ & $\mathrm{C}$ & $\mathrm{C}$ & $\mathrm{D}$ & $\mathrm{D}$ \\
$q_{1}(\mathrm{~mm})$ & 60.0 & 2.0 & 30.0 & 21.3 & 20.0 & 20.0 & 8.1 & 2.0 & 1.0 & 50.1 \\
$q_{2}\left(^{\circ}\right)$ & 1.0 & -16.7 & -17.1 & -19.3 & -18.3 & -7.6 & -13.2 & -14.1 & -16.0 & -1.1 \\
$q_{3}(\mathrm{~mm})$ & 66.6 & 49.5 & 60.0 & 22.4 & 21.2 & 20.0 & 63.5 & 61.9 & 60.3 & 58.8 \\
$q_{4}\left(^{\circ}\right)$ & 27.1 & 22.9 & 23.3 & 12.6 & -8.2 & -14.6 & 0.3 & -0.4 & 25.4 & 25.0 \\
$\begin{array}{c}\text { Insertion } \\
\text { depth (mm) }\end{array}$ & 43.7 & 38.8 & 43.8 & 47.1 & 41.9 & 29.9 & 28.8 & 28.5 & 42.0 & 41.6 \\
$\begin{array}{c}\text { In-plane } \\
\text { error (mm) }\end{array}$ & 1.1 & 2.9 & 1.7 & 3.2 & 4.8 & 3.3 & 4.5 & 3.2 & 1.5 & 0.5 \\
\hline \hline
\end{tabular}

Values refer to a set of 10 needle insertions with different directions, targeting 4 different points (ref. Fig. 14(b)). The in-plane error is calculated using 3D Slicer [56] as distance between center of the capsule and center of the needle artifact on the axial slice $(x y)$ at the center of the target. 


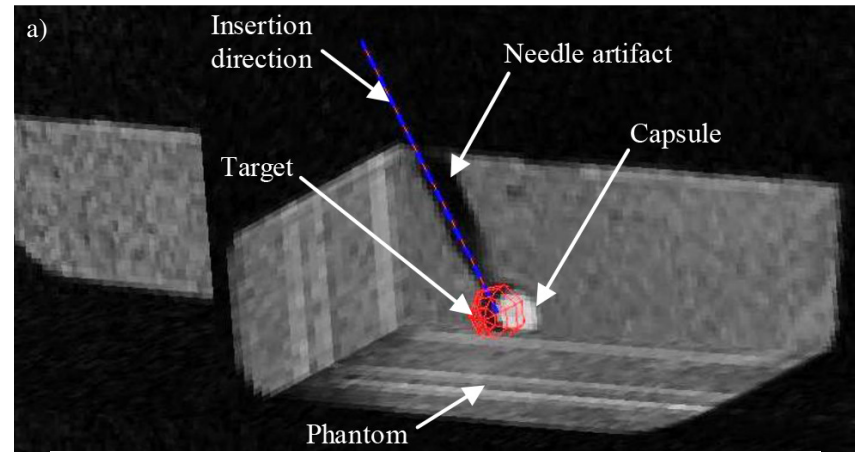

b)

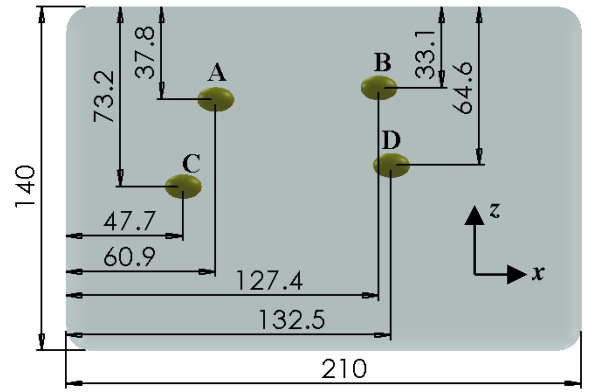

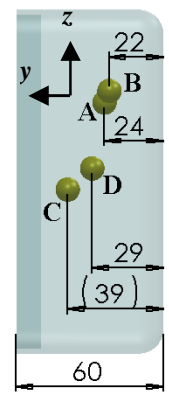

Fig. 14. Verification images for one test condition on the GUI (a): the red sphere is the target, which corresponds to the capsule (bright region); the blue dashed line is the needle axis; the needle artifact appears as a dark region. Schematic of the target positions within the phantom (b), dimensions in (mm).

\section{CONCLUSION}

We present a 4-DOF needle-guiding robot for MRI-guided LA of liver tumors. The robot was designed to operate inside a closed-bore MRI scanner in order to automatically align a needle guide to the target lesions, while still employing manual needle insertion outside the bore. The prototype is intended to operate within the established workflow of MRIguided LA, in which mechanical ventilation is employed during imaging and needle insertion to minimize liver motion.

The MRI-compatibility tests demonstrated that the system does not deteriorate the MR images with visible artifacts and does not cause noticeable reduction in SNR. The image uniformity analysis showed a limited variation of the indexes due to the presence of the robot. Due to the optical encoders employed, the current prototype qualifies as MRI-conditional.

For the position control of the robot we employed a TDC scheme designed with the backstepping technique. The comparison with a previously designed SMC algorithm showed that TDC is capable of higher steady-state accuracy but is less performing in tracking tasks. Additionally, TDC does not require assumptions on the magnitude of friction forces and external loads, which can be difficult to estimate with sufficient accuracy.

The phantom study conducted in a closed-bore MRI scanner suggests that the system is capable of targeting clinically relevant lesions. The potential time saving associated with the use of the needle-guiding robot in LA procedures is estimated to be 30 minutes for a single lesion. Due to the greater anatomic complexity of the human liver compared to the phantom used in this study and to the variability in size, number and location of the real lesions, this number is however only indicative.

In future work, we intend to compare targeting accuracy and duration between manual and robot-assisted needle insertion in the same conditions. Further tests on phantoms will be conducted in preparation for the clinical trials and a detailed analysis of the targeting error will be performed. Moreover, we intend to further improve the accuracy of the system, to introduce an additional depth gauge, and to reduce the registration time. Finally, we propose to investigate the fully automated needle insertion.

\section{ACKNOWLEDGMENT}

The authors are grateful to Karyn Chappell, for her assistance with the experiments in the MRI scanner, and to the anonymous reviewers, for their detailed and constructive comments that greatly improved this manuscript.

\section{REFERENCES}

[1] C. M. Pacella, G. Francica, and G. G. Di Costanzo, "Laser Ablation for Small Hepatocellular Carcinoma," Radiology Research and Practice, vol. 2011.pp. 1-8, 2011.

[2] J. Bruix and M. Sherman, "Management of hepatocellular carcinoma.," Hepatology, vol. 42, no. 5, pp. 1208-36, Nov. 2005.

[3] E. A. Dick, S. D. Taylor-Robinson, H. C. Thomas, and W. M. W. Gedroyc, "Ablative therapy for liver tumours.," Gut, vol. 50, no. 5, pp. 733-739, 2002.

[4] T. J. Vogl, P. K. Müller, R. Hammerstingl, N. Weinhold, M. G. Mack, C. Philipp, M. Deimling, J. Beuthan, W. Pegios, and H. Riess, "Malignant liver tumors treated with MR imaging-guided laser-induced thermotherapy: technique and prospective results.," Radiology, vol. 196, no. 1, pp. 257-265, 1995.

[5] A. L. Gough-Palmer and W. M. W. Gedroyc, "Laser ablation of hepatocellular carcinoma--a review.," World J. Gastroenterol., vol. 14, no. 47, pp. 7170-7174, 2008.

[6] W. M. W. Gedroyc, "Magnetic resonance guidance of thermal ablation.," Top. Magn. Reson. Imaging, vol. 16, no. 5, pp. 339-353, 2005.

[7] N. Hata, R. Hashimoto, J. Tokuda, and S. Morikawa, "Needle guiding robot for MR-guided microwave thermotherapy of liver tumor using motorized remote-center-of-motion constraint," in Proceedings - IEEE International Conference on Robotics and Automation, 2005, vol. 2005, pp. $1652-1656$.

[8] S.-E. Song, J. Tokuda, K. Tuncali, A. Yamada, M. Torabi, and N. Hata, "Design evaluation of a double ring RCM mechanism for robotic needle guidance in MRI-guided liver interventions," in 2013 IEEE/RSJ International Conference on Intelligent Robots and Systems, 2013, pp. 4078-4083.

[9] H. Elhawary, Z. T. Tse, A. Hamed, M. Rea, B. L. Davies, and M. U. Lamperth, "The case for MR-compatible robotics: a review of the state of the art," Int J Med Robot, vol. 4, no. 2, pp. 105-113, 2008.

[10] S. E. Song, J. Tokuda, K. Tuncali, C. M. Tempany, E. Zhang, and N. Hata, "Development and preliminary evaluation of a motorized needle guide template for MRI-guided targeted prostate biopsy," IEEE Trans. Biomed. Eng., vol. 60, no. 11, pp. 3019-3027, 2013.

[11] A. Krieger, S. E. Song, N. Bongjoon Cho, I. I. Iordachita, P. Guion, G. Fichtinger, and L. L. Whitcomb, "Development and evaluation of an actuated MRI-compatible robotic system for MRI-guided prostate intervention," in IEEE/ASME Transactions on Mechatronics, 2013, vol. 18 , no. 1, pp. 273-284.

[12] B. Yang, U. X. Tan, A. McMillan, R. Gullapalli, and J. P. Desai, "Design and implementation of a pneumatically-actuated robot for breast biopsy under continuous mri," in Proceedings - IEEE International Conference on Robotics and Automation, 2011, pp. 674-679.

[13] F. Sergi, A. C. Erwin, and M. K. OrMalley, "Interaction Control Capabilities of an MR-Compatible Compliant Actuator for Wrist Sensorimotor Protocols During fMRI," IEEE/ASME Trans. Mechatronics, vol. PP, no. 99, pp. 1-13, 2015.

[14] H. Su, W. Shang, G. Cole, G. Li, K. Harrington, A. Camilo, J. Tokuda, C. M. Tempany, N. Hata, and G. S. Fischer, "Piezoelectrically Actuated 
Robotic System for MRI-Guided Prostate Percutaneous Therapy," IEEE/ASME Trans. Mechatronics, vol. PP, no. 99, pp. 1-13, 2014.

[15] G. Li, H. Su, G. Cole, W. Shang, K. Harrington, A. Camilo, J. Pilitsis, and G. Fischer, "Robotic System for MRI-Guided Stereotactic Neurosurgery.," IEEE Trans. Biomed. Eng., vol. 62, no. 4, pp. $1077-$ 1088, Nov. 2014

[16] T. McPherson and J. Ueda, "A force and displacement self-sensing piezoelectric MRI-compatible tweezer end effector with an on-site calibration procedure," IEEE/ASME Trans. Mechatronics, vol. 19, no. 2 pp. 755-764, 2014.

[17] S. E. Song, N. B. Cho, G. Fischer, N. Hata, C. Tempany, G. Fichtinger, and I. Iordachita, "Development of a Pneumatic Robot for MRI-guided Transperineal Prostate Biopsy and Brachytherapy: New Approaches," IEEE Int Conf Robot Autom, vol. 2010, pp. 2580-2585, 2010.

[18] A. Melzer, B. Gutmann, T. Remmele, R. Wolf, A. Lukoscheck, M. Bock, H. Bardenheuer, and H. Fischer, "INNOMOTION for Percutaneous Image-Guided Interventions," Eng. Med. Biol. Mag. IEEE, vol. 27, no. 3 , pp. 66-73, 2008

[19] D. Stoianovici, C. Kim, G. Srimathveeravalli, P. Sebrecht, D. Petrisor, J Coleman, S. B. Solomon, and H. Hricak, "MRI-safe robot for endorectal prostate biopsy," IEEE/ASME Trans. Mechatronics, vol. 19, no. 4, pp. 1289-1299, 2014.

[20] S. Jiang, W. Feng, J. Lou, Z. Yang, J. Liu, and J. Yang, "Modelling and control of a five-degrees-of-freedom pneumatically actuated magnetic resonance-compatible robot," Int. J. Med. Robot. Comput. Assist. Surg., vol. 10 , no. 2 , pp. 170-179, 2014.

[21] B. Iranpanah, M. Chen, A. Patriciu, and S. Sirouspour, "A Pneumatically Actuated Target Stabilization Device for MRI-Guided Breast Biopsy," IEEE/ASME Trans. Mechatronics, vol. PP, no. 99, pp. 1-13, 2014.

[22] Y. Chen, C. D. Mershon, and Z. T. H. Tse, "A 10-mm MR-Conditiona Unidirectional Pneumatic Stepper Motor.," IEEE/ASME Trans. Mechatronics, vol. 20, no. 2, pp. 782-788, Apr. 2015.

[23] G. S. Fischer, I. Iordachita, C. Csoma, J. Tokuda, S. P. DiMaio, C. M Tempany, N. Hata, and G. Fichtinger, "MRI-Compatible Pneumatic Robot for Transperineal Prostate Needle Placement," IEEEASME Trans. Mechatronics, vol. 13, no. 3, pp. 295-305, 2008.

[24] B. Yang, U. X. Tan, A. McMillan, R. Gullapalli, and J. P. Desai, "Design and Control of a 1-DOF MRI Compatible Pneumatically Actuated Robot with Long Transmission Lines," IEEE ASME Trans Mechatron, vol. 16, no. 6 , pp. 1040-1048, 2011.

[25] E. Franco and M. Ristic, "Design and control of needle positioner for MRI-guided laser ablation of the liver," in 2014 IEEE/ASME 10th International Conference on Mechatronic and Embedded Systems and Applications (MESA), 2014, pp. 1-6.

[26] E. Franco and M. Ristic, "Time delay controller for the position control of a MRI-compatible pneumatic actuation with long supply lines," in 2014 IEEE/ASME International Conference on Advanced Intelligent Mechatronics, 2014, pp. 683-689.

[27] D. M. Kawamura, Diagnostic Medical Sonography: Abdomen and Superficial Structures. Philadelphia: Wilkins, Lippincott Williams \& 1997.

[28] M. D’Onofrio, R. De Robertis, E. Demozzi, S. Crosara, S. Canestrini, and R. Pozzi Mucelli, "Liver volumetry: Is imaging reliable? Personal experience and review of the literature.," World J. Radiol., vol. 6, no. 4 pp. 62-71, 2014

[29] ASTM F2503-13 Standard Practice for Marking Medical Devices and Other Items for Safety in the Magnetic Resonance Environment. ASTM International, 2013.

[30] Council Directive 93/42/EEC concerning Medical Devices machinery of the European Parliament and of the Council. EU: The European Parliament and the Council, 1993.

[31] Annex I, Essential health and safety requirements relating to the design and construction of machinery. EU: Directive 2006/42/EC on machinery of the European Parliament and of the Council, 2006.

[32] Annex I, Principal Elements of the Safety Objectives for Electrical Equipment Designed for Use within Certain Voltage Limits. EU: Voltage Directive (LVD) 2006/95/EC of the European Parliament and of the Council, 2006.

[33] G. I. Barbash and S. A. Glied, "New Technology and Health Care Costs - The Case of Robot-Assisted Surgery," N. Engl. J. Med., vol. 363, no. 8, pp. 701-704, 2010.

[34] K. Ahmed, A. Ibrahim, T. T. Wang, N. Khan, B. Challacombe, M. S Khan, and P. Dasgupta, "Assessing the cost effectiveness of robotics in urological surgery - A systematic review," BJU International, vol. 110, no. 10 . pp. $1544-1556,2012$
[35] C. K. Rowe, M. W. Pierce, K. C. Tecci, C. S. Houck, J. Mandell, A. B. Retik, and H. T. Nguyen, "A Comparative Direct Cost Analysis of Pediatric Urologic Robot-Assisted Laparoscopic Surgery Versus Open Surgery: Could Robot-Assisted Surgery Be Less Expensive?," Journal of Endourology, vol. 26, no. 7. pp. 871-877, 2012.

[36] E. G. Christoforou, I. Seimenis, E. Andreou, E. Eracleous, and N. V. Tsekos, "A novel, general-purpose, MR-compatible, manually actuated robotic manipulation system for minimally invasive interventions under direct MRI guidance,” Int. J. Med. Robot. Comput. Assist. Surg., vol. 10, no. 1, pp. 22-34, Mar. 2014.

[37] M. M. Arnolli, N. C. Hanumara, M. Franken, D. M. Brouwer, and I. A. M. J. Broeders, "An overview of systems for CT- and MRI-guided percutaneous needle placement in the thorax and abdomen.," Int. J. Med. Robot., Dec. 2014

[38] D. J. van Gerwen, J. Dankelman, and J. J. van den Dobbelsteen, "Needletissue interaction forces--a survey of experimental data.," Med. Eng. Phys., vol. 34, no. 6, pp. 665-80, 2012.

[39] N. Gulati and E. J. Barth, "A Globally Stable, Load-Independent Pressure Observer for the Servo Control of Pneumatic Actuators," Mechatronics, IEEE/ASME Trans., vol. 14, no. 3, pp. 295-306, 2009.

[40] ISO 6358:1989 - Pneumatic fluid power -- Components using compressible fluids -- Determination of flow-rate characteristics. ISO, 1989.

[41] E. Richer and Y. Hurmuzlu, "A High Performance Pneumatic Force Actuator System: Part I-Nonlinear Mathematical Model," J. Dyn. Syst Meas. Control, vol. 122, no. 3, p. 416, Sep. 2000.

[42] G. M. Bone and S. Ning, "Experimental comparison of position tracking control algorithms for pneumatic cylinder actuators," in IEEE/ASME Transactions on Mechatronics, 2007, vol. 12, no. 5, pp. 557-561.

[43] L. Ljung, System Identification - Theory For the User. Upper Saddle River, NJ, 1999.

[44] K. Youcef-Toumi and O. Ito, “A Time Delay Controller for Systems with Unknown Dynamics.” pp. 904-913, 1988.

[45] S. Hodgson, M. Tavakoli, M. T. Pham, and A. Leleve, "Nonlinear Discontinuous Dynamics Averaging and PWM-Based Sliding Control of Solenoid-Valve Pneumatic Actuators," IEEE/ASME Trans. Mechatronics, vol. 20, no. 2, pp. 876-888, Apr. 2015.

[46] M. Krstic, I. Kanellakopoulos, and P. Kokotovic, Nonlinear and Adaptive Control Design. Wiley, 1995, p. 576.

[47] M. A. Azman, A. A. M. Faudzi, M. O. Elnimair, O. F. Hikmat, and K. Osman, "P-Adaptive Neuro-Fuzzy and PD-Fuzzy controller design for position control of a modified single acting pneumatic cylinder," in 2013 IEEE/ASME International Conference on Advanced Intelligent Mechatronics, 2013, pp. 176-181.

[48] J.-Y. Lai and F.-C. Ou, "The Application of Time Delay Control to a Pneumatic Servo System.” pp. 233-234, 1992.

[49] J. Brujić, S. F. Edwards, D. V Grinev, I. Hopkinson, D. Brujić, and H. A. Makse, "3D bulk measurements of the force distribution in a compressed emulsion system.," Faraday Discuss., vol. 123, pp. 207-220; discussion 303-322, 419-421, 2003.

[50] F. Galassi, D. Brujic, M. Rea, N. Lambert, N. Desouza, and M. Ristic, "Fast and accurate localization of multiple RF markers for tracking in MRI-guided interventions," Magnetic Resonance Materials in Physics, Biology and Medicine, 2014.

[51] N. Garnov, G. Thörmer, R. Trampel, W. Gründer, T. Kahn, M. Moche, and H. Busse, "Suitability of miniature inductively coupled RF coils as MR-visible markers for clinical purposes," Medical Physics, vol. 38, no. 11. p. $6327,2011$.

[52] A. De Oliveira, J. Rauschenberg, D. Beyersdorff, W. Semmler, and M Bock, "Automatic passive tracking of an endorectal prostate biopsy device using phase-only cross-correlation," Magn. Reson. Med., vol. 59 , no. 5, pp. 1043-1050, 2008.

[53] J. R. Parker, Algorithms for Image Processing and Computer Vision, 2nd Editio. New York, Chichester, 1997.

[54] Determination of Signal-to-Noise Ratio (SNR) in Diagnostic Magnetic Resonance Imaging. NEMA Standard Publication MS 1-2008, 2008.

[55] Determination of Image Uniformity in Diagnostic Magnetic Resonance Imaging. NEMA Standard Publication MS 3-2008, 2008.

[56] S. Pieper, M. Halle, and R. Kikinis, "3D Slicer," in Biomedical Imaging: Nano to Macro, 2004. IEEE International Symposium on, 2004, pp. 632635 Vol. 1.

[57] D. W. Mcrobbie, E. A. Moore, M. J. Graves, and M. R. Prince, "Radiology MRI : From Picture to Proton," Radiology, p. 474, 2004. 